\title{
Technè
}

La science au service de l'histoire de l'art et de la

préservation des biens culturels

47 | 2019

Bernard Palissy : nouveaux regards sur la céramique française aux $X V I^{e}$ et $X V I^{e}$ siècles

\section{L'identification des moulages des coquilles fossiles et des organismes actuels des rustiques figulines : un apport naturaliste à la caractérisation des ateliers successifs de Palissy et de ses émules}

The identification of casts of fossil shells and existent organisms in rustic glazed earthenware: a naturalistic contribution to the particularity of the workshops of Palissy and his followers

Jean-Claude Plaziat

\section{OpenEdition}

Journals

Édition électronique

URL : http://journals.openedition.org/techne/1569

DOI : 10.4000/techne. 1569

ISSN : 2534-5168

Éditeur

C2RMF

Édition imprimée

Date de publication : 1 juin 2019

Pagination : 102-115

ISBN : 978-2-11-152830-7

ISSN : 1254-7867

Référence électronique

Jean-Claude Plaziat, « L'identification des moulages des coquilles fossiles et des organismes actuels des rustiques figulines : un apport naturaliste à la caractérisation des ateliers successifs de Palissy et de ses émules », Technè [En ligne], 47 | 2019, mis en ligne le 01 juin 2020, consulté le 23 juillet 2020. URL : http://journals.openedition.org/techne/1569; DOI : https://doi.org/10.4000/techne.1569

La revue Technè. La science au service de l'histoire de l'art et de la préservation des biens culturels est mise à disposition selon les termes de la Licence Creative Commons Attribution - Pas d'Utilisation Commerciale - Pas de Modification 4.0 International. 


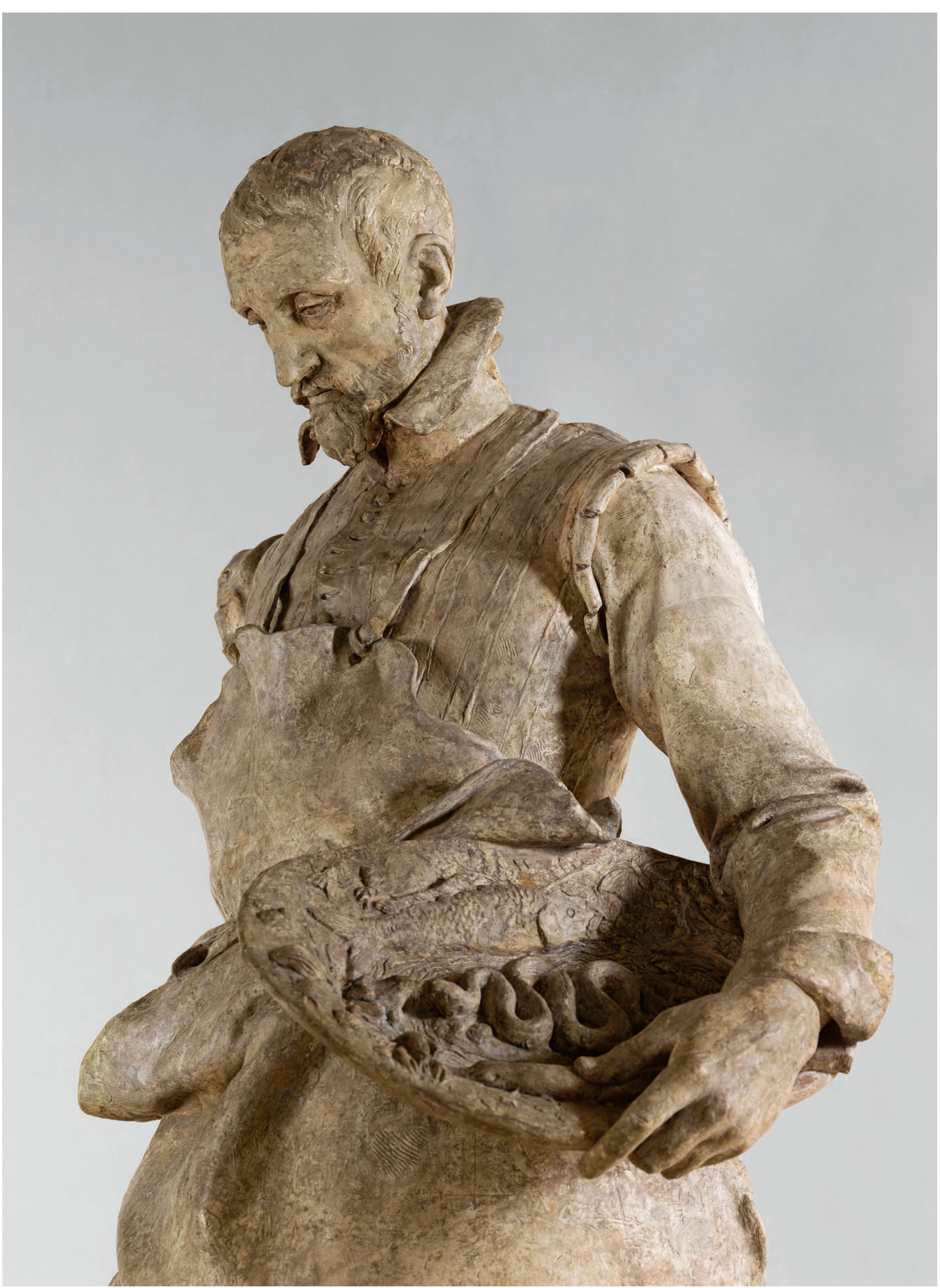

Fig. 1. Ernest Barrias, 1877, Bernard Palissy, plâtre (H. 215 cm, L. 102 cm, Pr. 92 cm), Paris, Petit Palais, musée des Beaux-Arts de la Ville de Paris, PPS8 (voir fig. 11). @ Petit Palais/Julien Vidal/Roger-Viollet. 


\section{Jean-Claude Plaziat L'identification des moulages des coquilles fossiles et des organismes actuels des rustiques figulines : un apport naturaliste à la caractérisation des ateliers successifs de Palissy et de ses émules}

The identification of casts of fossil shells and existent organisms in rustic glazed earthenware: a naturalistic contribution to the particularity of the workshops of Palissy and his followers

Résumé. Une révision de l'identification des organismes moulés (animaux actuels, fossiles du Tertiaire et végétaux) conduit à proposer une caractérisation des rustiques figulines produites par Bernard Palissy, à Saintes puis à Paris, par opposition à l'ensemble de la vaisselle à décor rustique due à différents émules post-palisséens anonymes, à partir de l'invention du décor «au serpent dans une île », qui est une véritable création artistique sans précédent. Celle-ci est caractérisée par des moulages de certaines coquilles fossiles de l'Éocène du nord du Bassin de Paris et de feuillages variés, ainsi que par le modelage d'insectes. Bien que nombreuses, les variantes de cette famille de plats à décor rustique suggèrent une production par des artisans successifs, dans un petit nombre d'ateliers, sans doute à partir du XVII siècle. Cette floraison se situe donc bien avant l'engouement du Romantisme pour l'artisanat d'art pré-classique qui a favorisé les céramistes néo-palisséens $d u$ XIX $X^{e}$ siècle, dont les æuvres n'ont abandonné que progressivement le moulage sur nature.

Mots-clés. Palissy, moulage sur nature, modelage, animaux marins, végétaux, fossiles, griffon, dragon, Tertiaire du Bassin parisien, Saintes, Paris, Sedan, Valois.
Abstract. A review of the identification of the different organisms cast from life (existent animals, Tertiary fossils and plants) led us to qualify the rustiques figulines produced by Bernard Palissy, first in Saintes, then Paris, as distinct from the dishes with rustic decoration made by various anonymous post-Palissian followers, after the invention of the "snake in an island" motif, which was an unprecedented artistic creation. This is characterised by casts made of certain Eocene fossil shells found in the northern Paris basin and of various insects and foliage. Although numerous, the variants of this type of rusticware suggest a series of craftsmen were involved in their production, in a small number of workshops, probably from the 17th century onwards. This flourishing output of glazed earthenware can thus be dated to well before Romanticism's love of medieval art which encouraged 19th-century neo-Palissian ceramists, whose works only gradually ceased to include life casts.

Keywords. Palissy, life cast, modelling, marine animals, plants, fossils, griffin, dragon, Paris Basin during the Tertiary, Saintes, Paris, Sedan, Valois.

\section{Introduction}

Bernard Palissy, céramiste exceptionnellement inventif, est aussi connu comme un savant naturaliste de premier plan, dont les écrits (Palissy, 1563, 1580) sont riches en découvertes et innovent dans bien des domaines ${ }^{1}$. Il est distingué, en particulier, depuis le Xvin ${ }^{\mathrm{e}}$ siècle, comme un véritable précurseur de la géologie scientifique, internationalement reconnu à ce titre par les pétrographes, les paléontologues et les pédologues d'aujourd'hui' ${ }^{2}$.

Sa magistrale interprétation de la nature des fossiles et ses commentaires sur l'origine environnementale des coquilles du Tertiaire de la Montagne de Reims n'ont pas manqué d'influencer les interprétations des premiers géologues (à partir de Brongniart) qui ont reconnu des fossiles du
Bassin parisien dans le décor d'un grand nombre de pièces appartenant à la vaisselle d'apparat qualifiée de rustiques figulines et globalement attribuée à Bernard Palissy ${ }^{3}$. La remarque vaut notamment pour les plats dont le décor s'organise autour d'un serpent dans une île (fig. 1) : une association de moulages sur nature de coquilles marines, actuelles et fossiles, entre des lézards, grenouilles et couleuvres dont Palissy a revendiqué l'introduction dans la décoration de céramiques.

Bien qu'elle ne prenne pas alors en considération les fossiles, Isabelle Perrin est la première à reconnaître cet ensemble, dont les caractéristiques stylistiques sont distinctes, comme entièrement dû à des céramistes postérieurs à Palissy ${ }^{4}$. Mais en les situant à la « fin du XVI ${ }^{\mathrm{e}}$ et au début du XvII ${ }^{\mathrm{e}}$ siècle", elle accrédite l'idée contestable de «continuateurs » ou de 


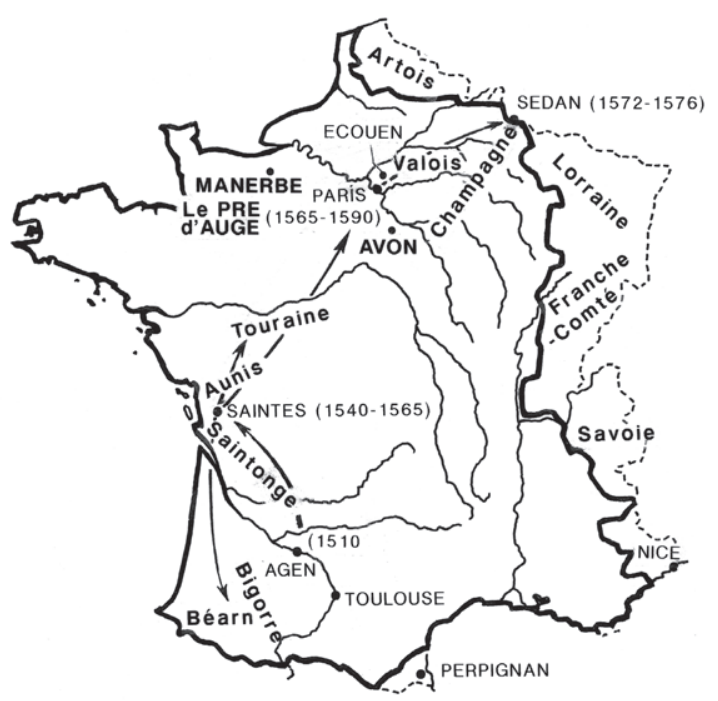

Fig. 2. Carte montrant les principaux déplacements de Bernard Palissy à l'intérieur du royaume de France. L'arrivée de Palissy à Saintes n'a pu être datée avec précision. (c) J.-C. Plaziat.

« suiveurs ", élèves directs et héritiers fidèles d'une « école palisséenne».

La révision de l'identification scientifique des organismes actuels et de ces fossiles, dont la provenance peut être établie sur des arguments géologiques régionaux, permet de renouveler aujourd'hui la conception typologique de cette vaisselle à décor rustique (palisséenne et post-palisséenne) et sa chronologie, jusqu'à étayer de nouvelles hypothèses concernant différents lieux de production ${ }^{5}$. La supposée transmission continue des conceptions de Palissy dans la décoration des plats rustiques créés postérieurement apparaît donc comme un malentendu qu'il s'agit de dissiper.

Cet article introduira d'abord le cadre historique de la production des rustiques figulines, qui s'échelonne sur près de cinq siècles ; il s'attachera ensuite à la description des sujets moulés caractéristiques de l'œuvre de Palissy, dans ses ateliers successifs de Saintes et de Paris (fig. 2) ; dans un second temps, il définira les particularités des décors rustiques de la vaisselle post-palisséenne et montrera en quoi ces nouveautés témoignent d'une rupture entre les créations de Palissy et celles des céramistes postérieurs.

\section{Le cadre historique de la production des diverses rustiques figulines $\left(\mathrm{XVI}^{\mathrm{e}}-\mathrm{XX}^{\mathrm{e}}\right.$ siècles $)$}

Entre l'invention des « rustiques figulines du Roy et de la Reine sa mère » revendiquée par Palissy à partir de 1563, et leur popularité renouvelée à partir du milieu du XIX ${ }^{\mathrm{e}}$ siècle, il s'est écoulé trois siècles pendant lesquels doit se situer la production de plats par ces céramistes anonymes qui ont inventé, adopté et développé le thème du "serpent dans une île ».

Le premier type de rustiques figulines, mis au point à Saintes, est l'aboutissement de recherches dont Palissy a abondamment souligné les difficultés, notamment concernant « les couleurs d'esmails» proches de celles de la nature ${ }^{6}$. Cette vaisselle au décor rustique inédit et à couverte polychrome sans précédent, dont les pièces d'apparat ont été admirées et collectionnées par Anne de Montmorency et par Henri II $^{7}$ peu avant le milieu du $\mathrm{Xvi}^{\mathrm{e}}$ siècle, correspond probablement à ce que les inventaires des biens parisiens du Connétable appellent la «façon de Xainctes ${ }^{8}$ ». Le décor moulé sur nature d'animaux et de plantes, que les orfèvres pratiquaient depuis longtemps ${ }^{9}$, semble donc avoir été introduit dans la pratique céramique, pour la première fois, par Palissy. En effet, l'attrait pour la représentation fidèle des créatures vivantes - l'un des thèmes apparus avec le maniérisme international - a rencontré, chez ce calviniste militant, la volonté de célébrer la Création divine, comme la Réforme le préconisait, à travers une figuration aussi fidèle que possible de la nature.

Ce décor par moulage de sujets naturalistes à l'échelle d'une vaisselle de prestige est ensuite éclipsé par une autre catégorie de rustiques figulines, que Palissy a imaginée pour constituer aussi un décor de «terre cuicte \& esmaillée ${ }^{10}$ » revêtant l'intérieur de grottes artificielles et de fontaines, ornements à la mode des jardins de l'aristocratie. Il vante jusqu'à l'excès, dans sa description publiée en 1563, le réalisme du décor de la grotte qu'il s'est engagé, en 1556, à réaliser pour le Connétable, dans l'atelier de Saintes. Une entreprise épuisante, abandonnée vers 1565, avant sa livraison, lorsqu'il est "invité » à se mettre au service de la reine mère, afin de réaliser, à Paris, la grotte à décor rustique dont elle veut orner les perspectives du jardin des Tuileries. Ces deux projets aux ambitions architectoniques démesurées pour un artisan particulièrement solitaire ont inévitablement absorbé une grande partie de son énergie et sans doute détourné Palissy de la production des bassins rustiques avant même qu'il ait quitté Saintes. En effet, dans l'ensemble considérable de tessons recueillis lors des fouilles autour du four des Tuileries, au Carrousel du Louvre, n'apparaît aucun des éléments (matrices ou contre-moulages) caractéristiques du vocabulaire nécessaire à la vaisselle rustique, en dehors d'exceptionnels vestiges inutilisables des plats produits à Saintes (le moule en plâtre d'un bassin et quelques fragments de plat non glaçuré).

Cependant, la renommée et la valeur des pièces de vaisselle d'apparat rustiques, dignes des collections royale et seigneuriale, ne s'étaient sans doute pas effacées des mémoires au début du XVII ${ }^{\mathrm{e}}$ siècle $^{11}$. C'est probablement bien après la mort de Palissy qu'apparaissent les fameux plats dont le décor rustique comporte un serpent dans une île. Leurs moulages de coquilles actuelles et fossiles constituent, en effet, une référence purement thématique aux éléments de la nature prisés par Palissy. Car l'intérêt qu'il a montré pour les fossiles du Tertiaire parisien n'est exprimé que tardivement (à partir de 1575), dans ses conférences publiques et dans son ouvrage publié en 1580. Il n'a jamais utilisé de fossiles dans son œuvre céramique ; ce qu'atteste l'absence totale de moulages de fossiles dans le matériel des fouilles du Carrousel du Louvre. 
La technique du moulage sur nature reste dominante parmi ces émules successifs, à l'exception de l'introduction très minoritaire de modelage ${ }^{12}$. Ce n'est que bien plus tardivement, lorsque se sont imposés les sujets en haut-relief nécessitant l'intervention d'habiles sculpteurs-modeleurs, que se développe une nouvelle conception de la vaisselle rustique, du XIX ${ }^{\mathrm{e}}$ au début du XXI ${ }^{\mathrm{e}}$ siècle. Des générations d'artistes que l'on peut qualifier de «néo-palisséens » privilégient désormais le modelage ${ }^{13}$, alors que certains, comme G. Pull et F. Maurice, sont restés fidèles au thème du serpent dans une île et à l'exclusivité du moulage sur nature. Ainsi ont-ils produit des plats qui pourraient être pris pour des contrefaçons des plats postpalisséens (s'ils n'étaient signés). Personne n'ayant eu l'idée de copier les rarissimes bassins rustiques de Palissy.

\section{Les sujets de moulages qui caractérisent les productions des ateliers successifs de Palissy}

\section{Palissy à Saintes}

Latelier de Bernard Palissy, dans le rempart de Saintes (Charente-Maritime), est celui de l'« invention » de cette vaisselle d'apparat, dont la maîtrise n'a été atteinte qu'après vingt ans d'effort, peu avant 1556. Le décor des bassins de la « façon de Xainctes » illustre la nature de la Saintonge, à la fois maritime et terrestre. Il se caractérise par la fidèle représentation des serpents, tortues, lézards et grenouilles, obtenue grâce aux moulages sur nature de ces sujets, dont Palissy fait état dans ses écrits. Mais les coquillages et les poissons de l'océan tout proche sont également nombreux et variés. Ce sont d'abord les mollusques bivalves : coques et bucardes ${ }^{14}$, pétoncle ${ }^{15}$, mais aussi la praire ${ }^{16}$; et les gastéropodes (buccin, nasse, natice et murex) qui sont disponibles sur la côte rocheuse et sableuse des Charentes (voir tableau des espèces) ${ }^{17}$. Sur les bassins des musées de Lyon (fig. 3) et du Louvre (fig. 4) ${ }^{18}$, le crabe vert, un grondin et une raie juvénile sont mêlés aux petits vertébrés continentaux parmi lesquels on note la présence de cistudes (une tortue d'eau douce) et de pelotons de couleuvre à collier et de vipère aspic figurant dans l'axe des plats ${ }^{19}$, tandis que des couleuvres ondulantes sont placées à la périphérie. Le lézard vert, la grenouille verte, l'écrevisse à pattes rouges et divers poissons non identifiés complètent cet assemblage représentatif des divers milieux de vie de la Saintonge, mais dont la réunion est contraire à toute réalité écologique. Un autre trait caractéristique de la "main » de Palissy est la superposition partielle de plusieurs valves plates de pétoncles (deux à quatre $)^{20}$ (fig. 5). Il faut également noter une quasiabsence de feuillages sur ses pièces de vaisselle (une seule fougère), puisque les masses vertes d'aspect spongieux sont des artefacts et non les moulages d'une véritable mousse.

De la vaisselle créée ici par Palissy ne sont parvenus jusqu'à nous que cinq plats (dont deux dans des collections privées) et quatre pichets et aiguières ${ }^{21}$. Cette rareté paraît s'expliquer par une production très limitée, interrompue par le travail exténuant exigé pour la fabrication du décor de la

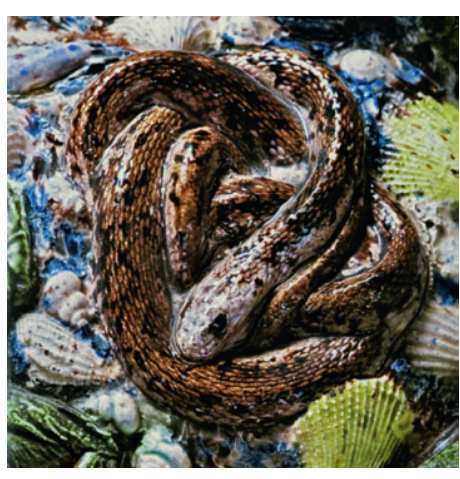

Fig. 3. Bernard Palissy, Détail d'un plat à décor de rustiques figulines : "nœud " de couleuvre à collier, Lyon, musée des Beaux-Arts, H 475. (C) J.-C. Plaziat.
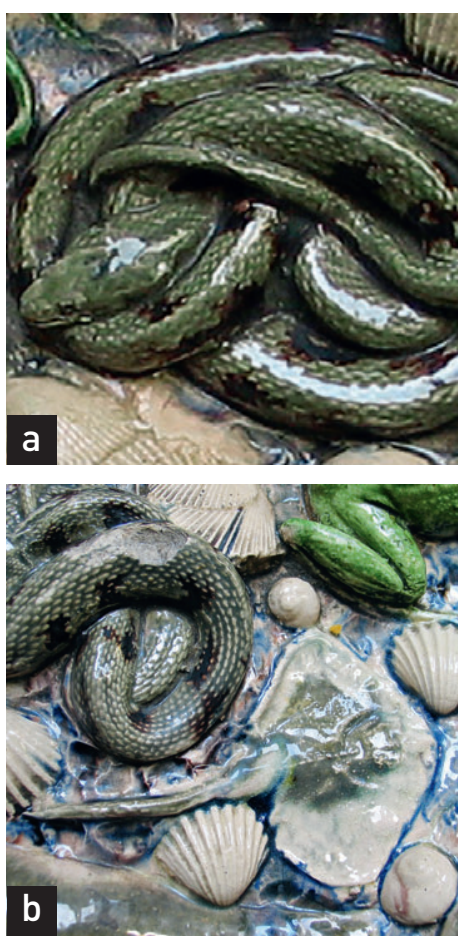

Fig. 4. Bernard Palissy, Détails d'un plat à décor de rustiques figulines, Paris, musée du Louvre, MR 2295. a. Couleuvre à collier ; b. Raie juvénile c. Tortue d'eau douce, lézards verts, grenouilles vertes, poissons marins, crabe enragé, entourés de coquilles du rivage de l'Atlantique (coques, bucardes, pétoncles, buccins et natice). (C) J.-C. Plaziat.
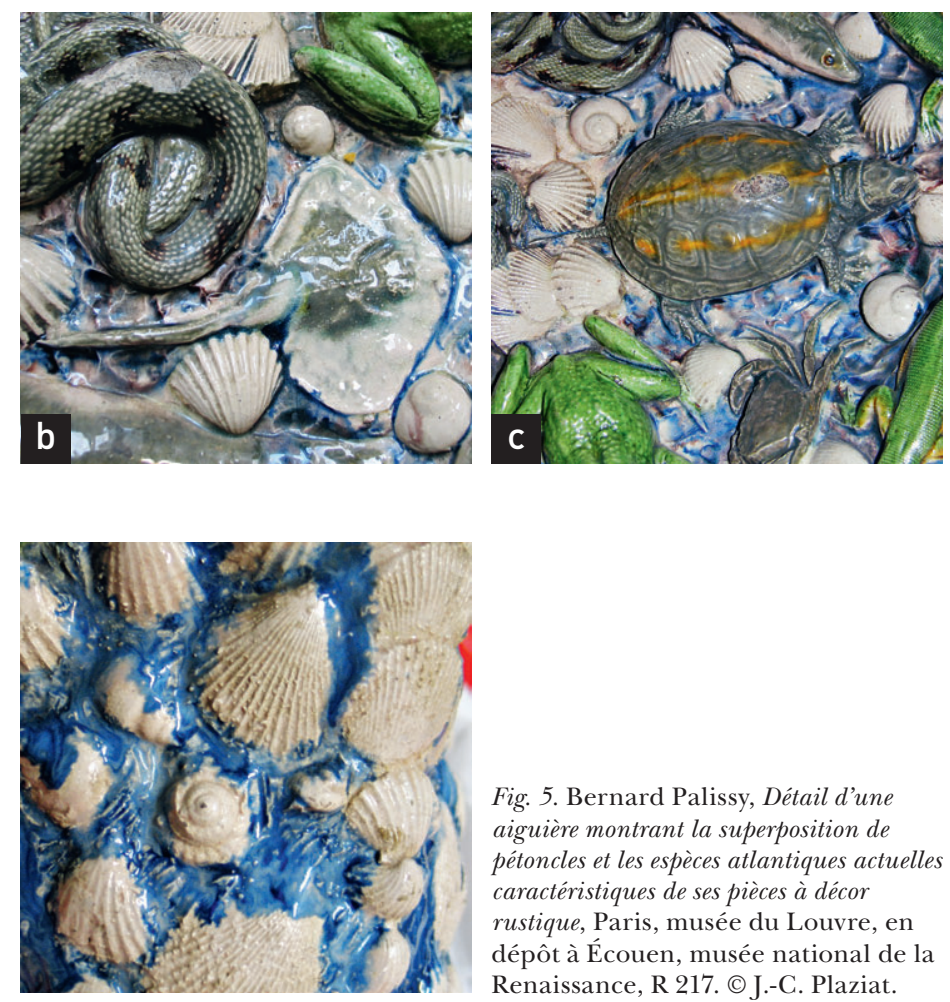

Fig. 5. Bernard Palissy, Détail d'une aiguière montrant la superposition de pétoncles et les espèces atlantiques actuelles caractéristiques de ses pièces à décor rustique, Paris, musée du Louvre, en dépôt à Écouen, musée national de la Renaissance, R 217. (C) J.-C. Plaziat. 


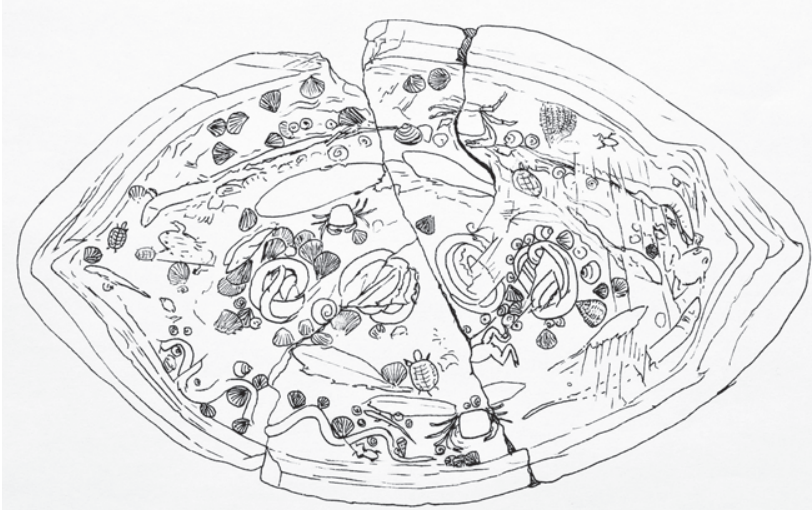

Fig. 6. Relevé graphique du moule de plat en plâtre trouvé dans l'atelier des Tuileries de Bernard Palissy, Écouen, musée national de la Renaissance, EP 3434 (extrait de Plaziat, 2011). (C) J.-C. Plaziat.

grotte du Connétable, dès 1556, et par l'exploitation de ses précédentes innovations techniques plus rentables, notamment la vaisselle « de terres mêlées ». Ces différents travaux ont nécessairement réduit le temps susceptible d'être consacré à cette production exigeante, pendant les dix années qui ont précédé son départ pour Paris.

\section{Palissy à Paris}

L'atelier situé sur le chantier des Tuileries, où Palissy a travaillé de 1566 ou 1567 à 1572, ne semble pas avoir été le lieu de fabrication d'une vaisselle de prestige à décor rustique, les monuments décoratifs du jardin étant, à nouveau, sa priorité. Cependant, il faut noter que toutes les particularités qui définissent sa production de Saintes se retrouvent sur un moule en plâtre d'un bassin exceptionnellement grand et imparfaitement démoulé, qui a été retrouvé près du four des Tuileries (fig. 6 et 7 a-f). Parmi les fragments de plat en terre non glaçurée, celui (fig. 8) qui reproduit à l'identique le décor animalier (lézard, jeune écrevisse et praire) d'une partie du plat du musée de Lyon (fig. 9) joue un rôle déterminant dans la caractérisation du décor palisséen. Ces découvertes archéologiques, dont le moulage de guêpe (avec l'inscription « fossalou - guepe ») et un moulage de phoque ${ }^{22}$, impliquent que Palissy a dû déménager une partie de son fonds d'atelier de Saintes, en privilégiant les éléments susceptibles d'être réutilisés pour la grotte de Catherine de Médicis, ainsi que quelques témoins de ses premières recherches abouties, mais dont il avait abandonné l'exploitation : celles qui nécessitaient les matrices d'animaux marins de petite taille (praire, poissons, crabe). Cela confirme, pour nous, l'hypothèse qu'il avait délaissé la production de sa vaisselle rustique bien avant de partir pour Paris. En revanche, le transfert du phoque, comme la présence de grands lézards et couleuvres, de coques et de buccins de grande taille, qui figurent aussi sur les nombreux restes fragmentaires du décor destiné à la grotte des Tuileries,
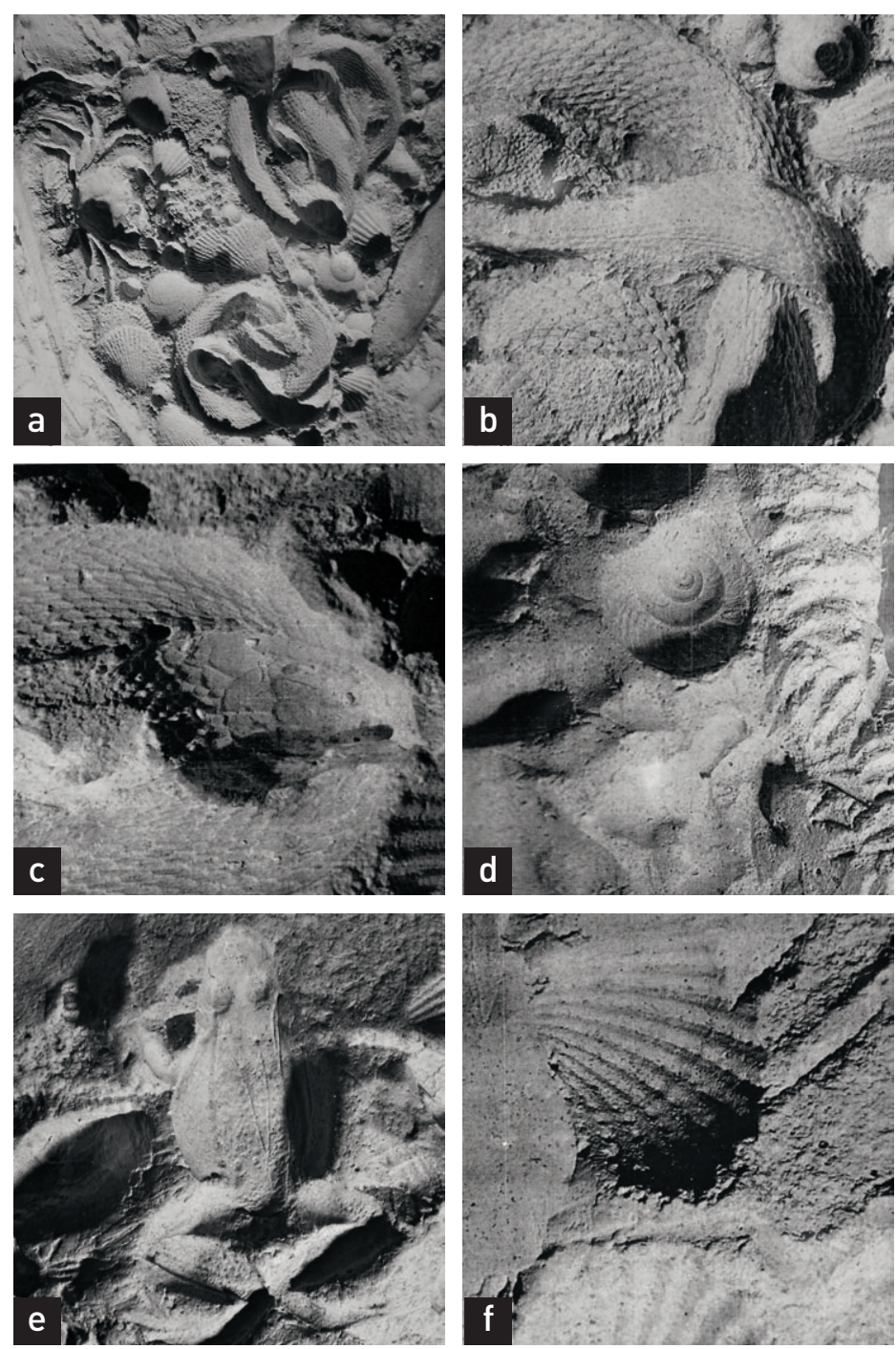

Fig. 7. Atelier de Bernard Palissy, Paris, fouilles des Tuileries, Détails (en lumière rasante et retournement du cliché donnant l'effet en positif) du moule de plat à décor de rustiques figulines, plâtre, Écouen, musée national de la Renaissance, EP 3434. a. Décor axial de droite : crabe, pétoncles se chevauchant, peloton de couleuvre ; b. Nœud de vipère identifiable par les petites écailles sur la tête ; c. Couleuvre ; d. Petit escargot ; e. Grenouille ; f. Bucarde. ( J.-C. Plaziat.

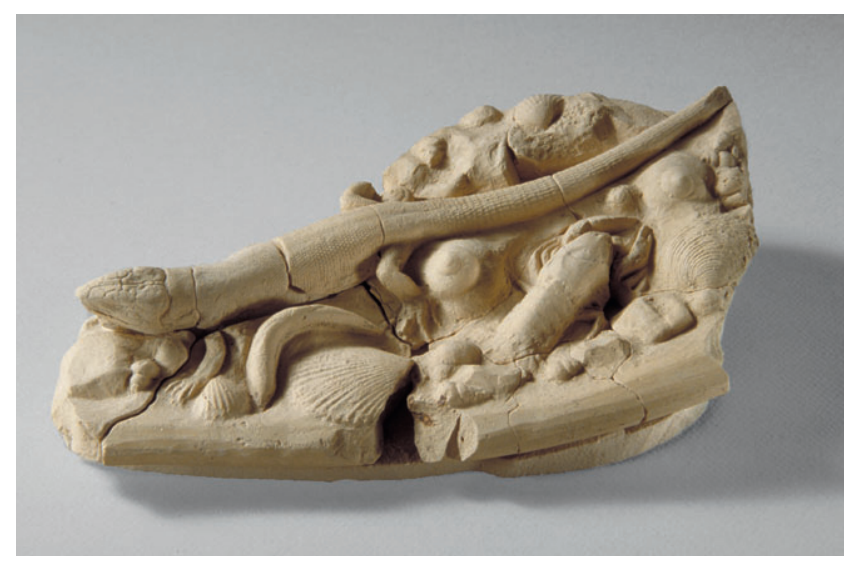

Fig. 8. Atelier de Bernard Palissy, Paris, fouilles des Tuileries, Fragment de plat à décor de rustiques figulines, terre cuite $(\mathrm{L} .25 \mathrm{~cm}, 1.14,5 \mathrm{~cm}$, H. 4 cm), Paris, musée du Louvre, Histoire du Louvre, JC 12123946 (ENT 1999.51) (C) Musée du Louvre, Dist. RMN-Grand Palais/Pierre Philibert. 


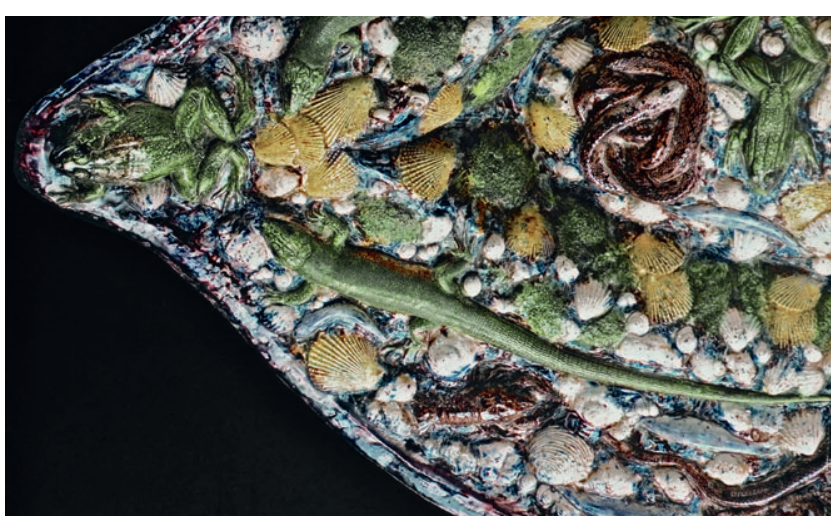

Fig. 9. Bernard Palissy, Détail de la partie correspondante au fragment de la fig. 8 d'un plat à décor de rustiques figulines, Lyon, musée des Beaux-Arts, H 475. () J.-C. Plaziat.
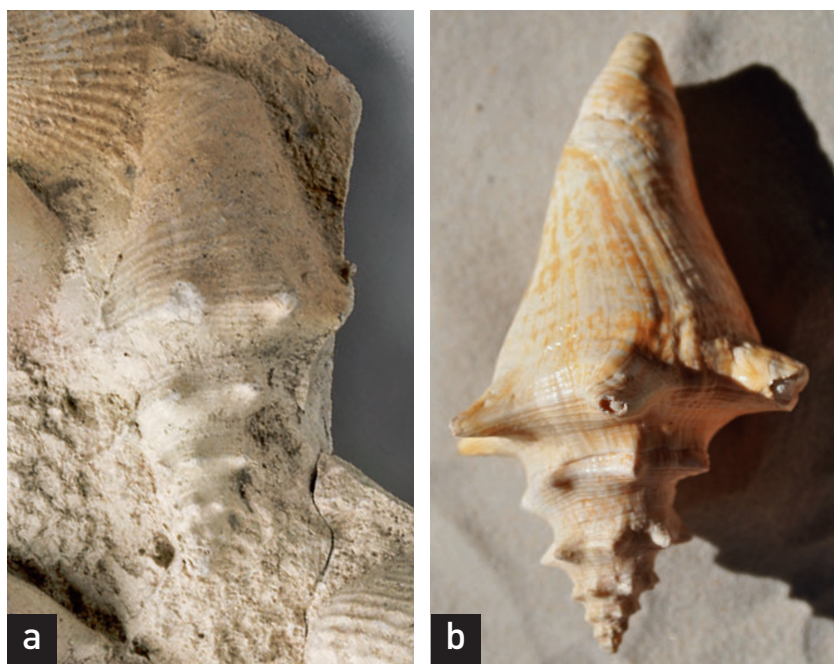

Fig. 10. a. Atelier de Bernard Palissy, Paris, fouilles des Tuileries, Détail de strombe géant des Antilles (Strombus gigas) sur un fragment de terme, plâtre, Écouen, musée national de la Renaissance, EP 1291. (C) RMN-Grand Palais (musée national de la Renaissance, château d'Écouen)/René-Gabriel Ojéda ; b. Un spécimen juvénile de cette espèce. (C) J.-C. Plaziat.

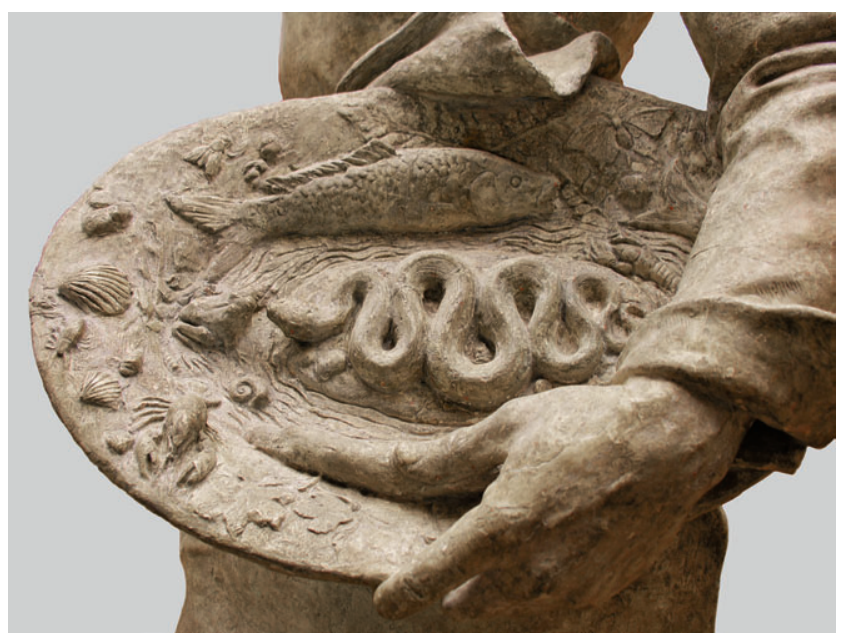

Fig. 11. Un exemple de la conception erronée des rustiques figulines attribuées à Palissy jusqu'à la fin du $\mathrm{xx}^{\mathrm{e}}$ siècle. Détail du plat de la fig. 1. () J.-C. Plaziat. s'explique par la nécessité de tenir compte de l'échelle architectonique monumentale des édifices commandés. De même, de grandes coquilles Saint-Jacques et l'escargot de Bourgogne font ici leur apparition. Mais c'est le strombe géant des Antilles qui est le plus intéressant (fig. 10) ${ }^{23}$. L'acquisition de cette espèce exotique suppose des contacts avec le milieu des découvreurs français de l'outre-mer, souvent calvinistes comme Palissy, qu'il n'a vraisemblablement pu rencontrer qu'à Paris, entre 1566 et 1572, car les marins des ports d'Aquitaine étaient alors moins entreprenants que ceux de la Manche, en relation avec la cour.

Lorsqu'il séjourne à Sedan, à partir de 1572, après avoir échappé au massacre de la Saint-Barthélemy, Palissy ne semble pas avoir repris une activité de production de vaisselle rustique dans son nouvel atelier de céramiste, partagé avec son gendre puis délaissé avant 1584. De retour à Paris dès 1575 , il se consacre à l'activité intellectuelle de savant couronnée par la publication de son principal ouvrage, en 1580, qui assure sa gloire mais le conduira vers un destin funeste. Quand il meurt à la Bastille, en 1590 selon Pierre de l'Étoile, on peut donc admettre qu'il n’a plus produit les emblématiques plats à décor rustique depuis plus d'une trentaine d'années.

\section{La vaisselle d'apparat dite post-palisséenne}

L'ensemble considérable des rustiques figulines, qui correspondent à la vaisselle anonyme aujourd'hui retirée du corpus palisséen ${ }^{24}$, est surtout connu par les plats dont le décor s'organise autour d'une île ovale (avec ou sans couleuvre axiale), entourée d'un anneau d'eau plus ou moins turbulente (avec ou sans poissons, tous d'eau douce). C'est le type de décor le plus emblématique, avec ses variantes, qui a été retenu dans toutes les représentations de Palissy célébrant sa gloire par les statues officielles du Xıx ${ }^{\mathrm{e}}$ siècle (fig. 1 et 11) et dans les illustrations didactiques jusqu'au milieu du siècle dernier ; comme dans le choix des plats de musées supposés illustrer l'œuvre du maître dans la plupart des ouvrages de vulgarisation, jusqu'à aujourd'hui' ${ }^{25}$.

Contrairement aux pièces relevant des ateliers de Bernard Palissy, les céramiques à décor rustique post-palisséennes ne bénéficient pas, jusqu'à présent, de données chronologiques et géographiques incontestables. Elles ont d'ailleurs été datées aussi bien de la fin du Xvi ${ }^{\mathrm{e}}$ siècle que du début $\mathrm{du} \mathrm{XIX}^{\mathrm{e}}$ siècle, selon les auteurs, et attribuées à des ateliers divers, en particulier d'Avon (Fontainebleau) ou de Normandie (Pré-d'Auge) ${ }^{26}$. Cependant, des jalons chronologiques d'apparition dans des collections ont été introduits récemment dans ce corpus, grâce aux études historiques réalisées sur la provenance des céramiques à décor rustique des musées d'Amiens, du Louvre et de Rennes ${ }^{27}$, dont l'acquisition pourrait remonter au XVII ${ }^{\mathrm{e}}$ siècle ou au début du XVIII ${ }^{\mathrm{e}}$. Un certain nombre de ces céramiques peut désormais être considéré comme des repères fiables, applicables aux regroupements typologiques proposés. 


\section{Caractérisation générale des décors rustiques post-palisséens}

On observe d'abord un changement dans la symétrie générale des plats : on ne retrouve plus dans les décors post-palisséens l'obsession de symétrie bilatérale qui caractérise ceux de Palissy. Les inévitables lézards verts, grenouilles et grosses écrevisses occupent presque uniquement le domaine émergé de l'aile, disposés de façon plus aléatoire que chez Palissy, combinés avec un riche échantillonnage de feuillages (fougères et plantes terrestres) qui rayonnent sur l'aile, alternant avec les coquilles de mollusques marins, actuels et fossiles, semées dans les espaces laissés libres entre les animaux terrestres et amphibies et les végétaux. L'organisation rayonnante des végétaux, l'absence de la praire, du buccin et des superpositions de pétoncles, qui caractérisent les œuvres de Palissy, sont à souligner. Mais c'est la présence de coquilles de gastéropodes fossiles, associés aux coques modernes et à un choix nouveau et extrêmement variable de gastéropodes actuels (parfois exotiques), qui semble la nouveauté la plus déterminante. Des fossiles qui ont attiré l'attention de rares paléontologues et qui n'ont été pris en compte que tardivement ${ }^{28}$.

Le choix de ces fossiles s'est porté principalement sur des gastéropodes dont les coquilles parfaitement conservées proviennent des sables marins de l'Éocène du Bassin parisien. Leur taille, leur forme et leur ornementation étant compatibles avec l'espace réservé et avec une mise en valeur par la lumière. Les bivalves fossiles sont moins variés et plus rarement utilisés ${ }^{29}$ mais ils proviennent, à l'évidence, des mêmes niveaux géologiques. Ces fossiles sont aussi parfaits que des coquilles actuelles, bien qu'ils aient vécu au Cuisien (il y a 50 millions d'années) et au Lutétien (il y a 45 millions d'années) dans le golfe de la mer du Nord qui a recouvert à plusieurs reprises le centre du Bassin parisien (voir tableau des espèces $)^{30}$. Ils proviennent nécessairement des affleurements et des modestes exploitations de sables qui étaient nombreuses aux siècles derniers entre Versailles (Grignon) et Reims (Damery) pour le Lutétien (Éocène moyen), et au nord de Paris (entre Compiègne et Soissons) pour ce qui

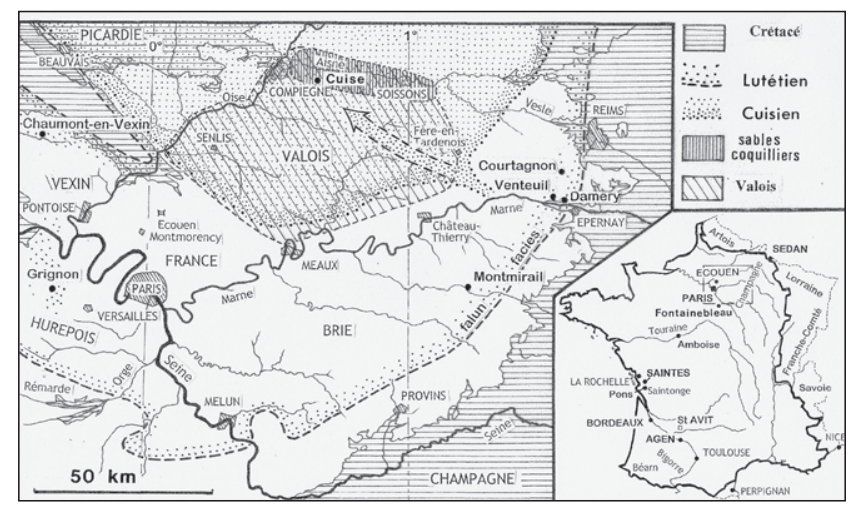

Fig. 12. Carte du centre du Bassin parisien montrant les zones géologiques d'où doivent provenir les coquilles fossiles de l'Éocène (extraite de Plaziat, 2009). () J.-C. Plaziat.

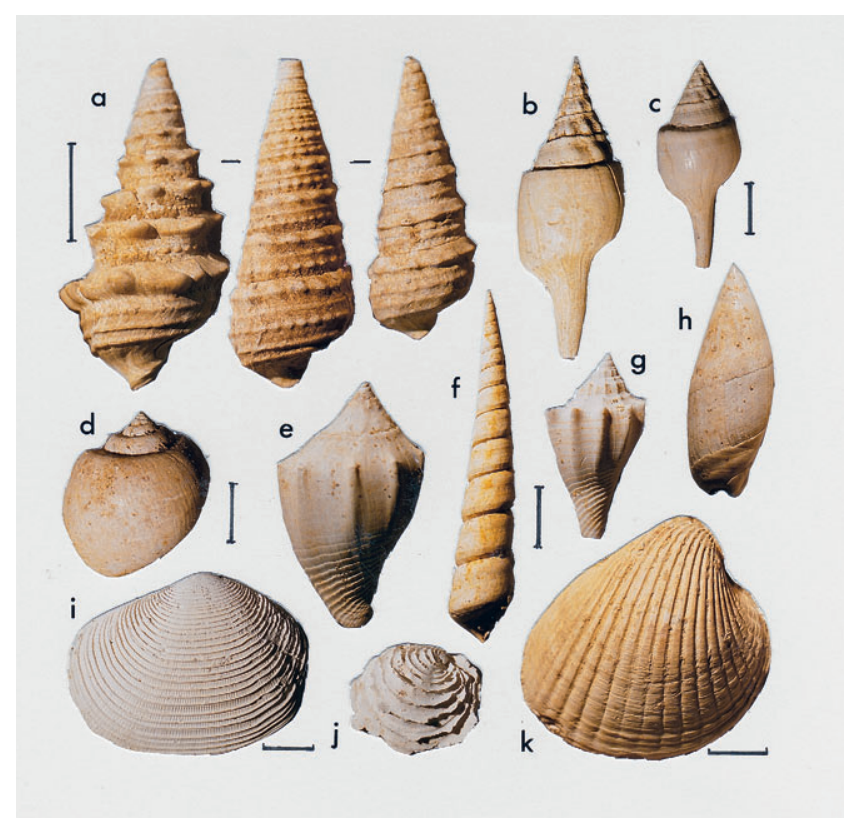

Fig. 13. Les coquilles des sables marins du Tertiaire, dont les moulages ont servi de matrice aux décors rustiques post-palisséens ( $\mathrm{C}=$ Cuisien ; $\mathrm{L}=$ Lutétien $)$. a : Eotympanotonus turris - funatus (potamides du Cuisien), b : Clavilithes parisiensis subscalaris (C), c : Clavilithes parisiensis (Lut.), d : Crommium cf. intermedia (C), e : Athleta depressa (C), f: Haustator solanderi (= Turritella edita) (C), $\mathrm{g}:$ Athleta spinosus (L), h : Ancilla buccinoides (Cuiso-Lut), i : Fimbria lamellosa $(\mathrm{C}-\mathrm{L}), \mathrm{j}$ : Chama punctata $(=$ C. calcarata $)(\mathrm{L}), \mathrm{k}:$ Venericor planicosta (petite forme cuisienne). Échelles graphiques : $1 \mathrm{~cm}$. (C) J.-C. Plaziat.

concerne le Cuisien (Yprésien marin, Éocène inférieur) (fig. 12). La plus grande partie des espèces, dont la durée d'existence fut relativement longue, peuvent être aussi bien lutétiennes que cuisiennes, mais certaines sont caractéristiques du Lutétien et d'autres du Cuisien. Et l'association de ces dernières ne peut provenir que d'un dépôt très particulier, affleurant uniquement aux environs de Cuise-la-Motte (près de Pierrefonds, Oise) : un dépôt de sable coquillier qui correspond au remplissage d'un paléo-chenal sous-marin, creusé dans le prolongement de l'estuaire d'un fleuve champenois. Ce qui explique un transport considérable de coquilles lors de crues majeures, qui a réuni des mollusques vivant en milieu estuarien ${ }^{31}$ avec des espèces strictement marines $^{32}$ (fig. 13 et 14).

\section{La question de l'atelier du Maître du dragon : nouvelles hypothèses}

La prise en compte de ces fossiles concerne particulièrement le groupe de plats au serpent dans l'île dit du « Maître du griffon » (une dénomination qu'il faut corriger en « Maître du dragon » car il ne s'agit pas d'une chimère d'oiseau). C'est Léonard Amico qui a proposé de regrouper sous ce nom erroné les plats présentant, sur l'aile, une petite figure de dragon en relief (moulage d'orfèvrerie) : le plat du Louvre 

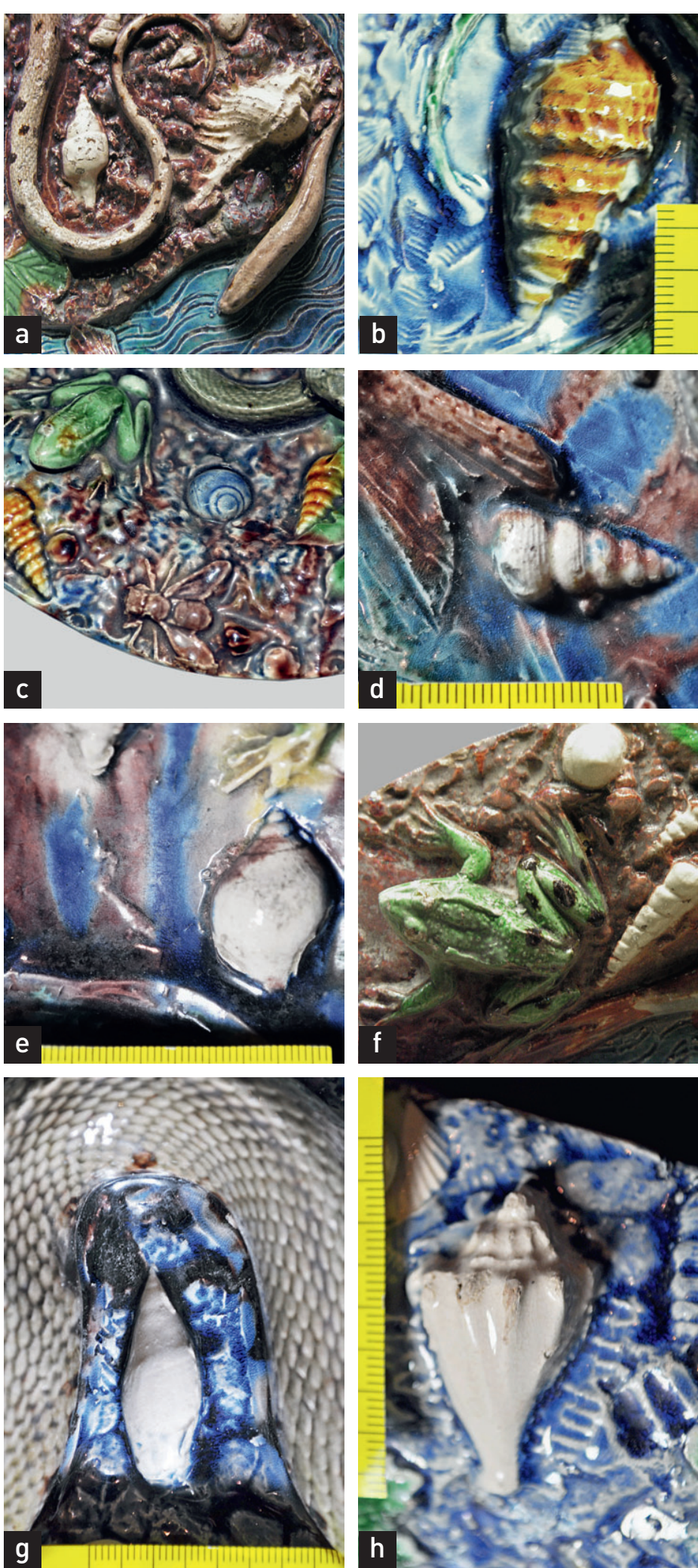

Fig. 14. Céramiques post-palisséennes : quelques incorporations de fossiles représentatifs du Cuisien et du Lutétien du Bassin parisien, à côté de coquilles marines actuelles.

a. Murex exotique et Clavilithes subscalaris dans la boucle de la queue d'une couleuvre, Paris, musée du Louvre, MR 2294. (c) J.-C. Plaziat. b. Potamide caractéristique du Cuisien (Eotympanotonus turris), Rennes, musée des Beaux-Arts, 794.1.569. (C) J.-C. Plaziat.

c. Association de moulages de potamides du Cuisien et d'une volute lutétienne, avec une grenouille, une couleuvre, des feuilles (toutes moulées), et une mouche-abeille géante (modelée), Rennes, musée des Beaux-Arts, 794.1.569. (C) J.-C. Plaziat.

d. Sigmesalia (C-L) moulé et queue de poisson fortement retouchée, Paris, musée du Louvre, MR 3530. (C) J.-C. Plaziat.

e. Sycostoma (C-L) et patte de grenouille moulés, Paris, musée du Louvre, MR 3530. (C) J.-C. Plaziat.

f. Haustator solanderi $(\mathrm{C})$, un potamide plus petit, non identifié, un poisson et une grenouille verte avec les taches noires caractéristiques sur l'arrière-train (tous fidèlement moulés), Paris, musée du Louvre, MR 2294. (c) J.-C. Plaziat.

g. Ancilla buccinoides (C-L) dans l'ondulation d'une couleuvre parfaitement moulée, Paris, musée du Louvre, MR 2293.

(C) J.-C. Plaziat.

h. Athleta spinosus (L) en fort relief et moulages en creux (négatifs) d'autres coquilles qui ornent le fond bleu, Paris, musée du Louvre, MR 2293. ( ) J.-C. Plaziat.

OA 1361 en étant l'œuvre référence d'un ensemble ${ }^{33}$ où le dragon peut être remplacé, par exemple par un serpent enroulé en spirale (fig. 15). Certaines pièces de cette série comportent non seulement le même décor végétal (feuilles de fougère et de lierre), mais aussi les mêmes fossiles du Lutétien et du Cuisien, associés à des insectes modelés mais pas systématiquement au dragon. En ce qui concerne l'identification des espèces de fossiles caractéristiques du Cuisien, la médiocre qualité du moulage de leurs coquilles sur les plats qui comportent le dragon nous a incité à nous tourner vers d'autres pièces de cet ensemble ${ }^{34}$, notamment celles où apparaît le chabot (poisson de rivière dont la grosse tête l'a souvent fait confondre avec un grondin marin, fig. 16), en plus du brochet et des autres poissons d'eau douce fidèlement moulés ${ }^{35}$.

Ce groupe comporte de multiples variantes, mais un dénominateur commun dans les moulages de végétaux et les modelages d'insectes très divers (papillon, petit hanneton, libellule, fig. 17). Ainsi, l'introduction des taches noires sur l'arrièretrain des grenouilles vertes, une source vive qui a pu être oblitérée par la superposition d'une grenouille, la sélection d'écrevisses de taille exceptionnellement grande, ou encore l'innovation des « galets » dispersés dans l'anneau d'eau vive ${ }^{36}$, avec ou sans poissons, et associée ou non à celle de la source, pourraient passer pour des créations indépendantes. Ces caractéristiques relèvent pourtant bien du vocabulaire de cet atelier du « Maître du dragon » qui a inséré la même libellule modelée (dont les nervures imaginaires des ailes sont caractéristiques), une grosse chenille et divers coléoptères modelés, associés aux grenouilles tachetées et aux fossiles du Cuisien.

L'intérêt chronologique de cet élargissement considérable du groupe du « Maître du griffon », tel que défini par Amico, a été évidemment décuplé lorsqu'il est apparu que deux des pièces du Louvre, qui peuvent y être rattachées, proviennent des saisies napoléoniennes opérées dans les 


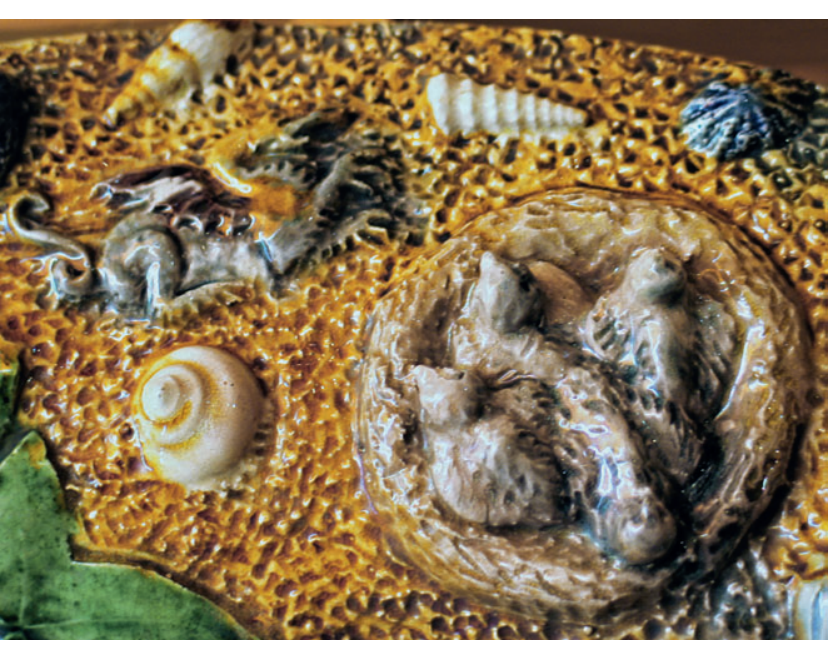

110

Fig. 15. Céramique post-palisséenne : détails du dragon et du nid d'oiseau miniaturisés (moulages de pièces d'orfèvrerie), associés à un Potamides cristatus, à une Ampullariidae (fossiles du Lutétien) et à une patelle actuelle (en haut, à dr.), sur le plat éponyme de l'ensemble attribué à l'« atelier du Maître du griffon » par Léonard Amico, Paris, musée du Louvre, OA 1361. ( J.-C. Plaziat.
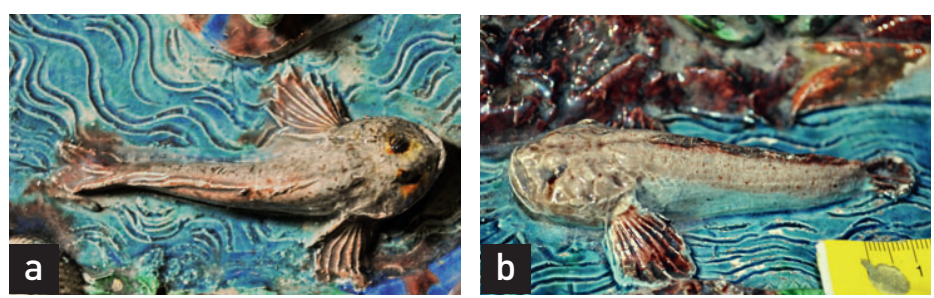

Fig. 16. Céramiques post-palisséennes (a et b) : deux exemples de chabot (Cottus), poisson des ruisseaux turbulents, Paris, musée du Louvre, MR 3530 et MR 2294. () J.-C. Plaziat.
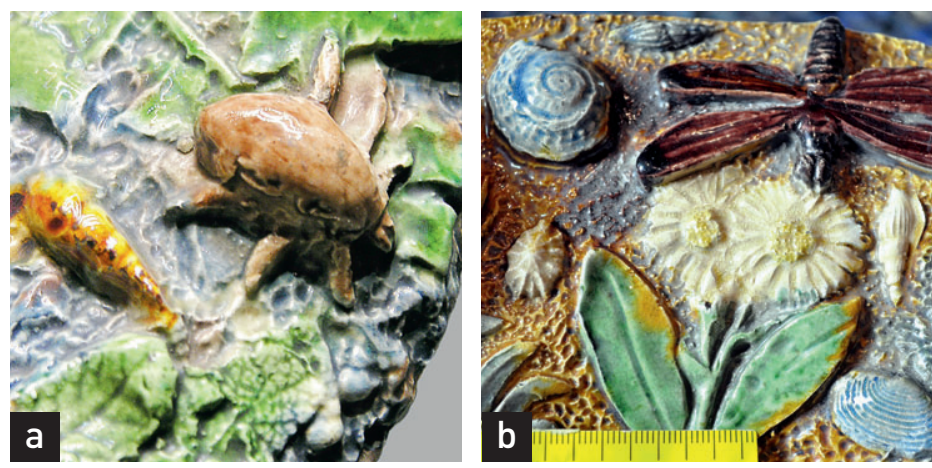

Fig. 17. Céramique post-palisséenne : exemples d'insectes modelés en partie ou en totalité, Paris, musée du Louvre, OA 1361.

a. Un coléoptère non identifiable. b. Une libellule associée aux fleurettes, et à des fossiles moulés (Rimella, à dr. des fleurs ; Fimbria, au-dessous), ainsi qu'à la petite patelle actuelle (à g.). @ J J.-C. Plaziat. collections des musées de Brunswick et de Cassel $^{37}$. Et surtout, lorsqu'a été reconnu le dessin d'un plat disparu de l'ancienne collection conservée au musée d'Amiens, qui est à associer indéniablement aux productions dites du « Maître du dragon » (fig. 18).

\section{Les décors rustiques des pièces historiques de référence}

La découverte de moulages de fossiles propres au faciès de Cuise-la-Motte m'a conduit à proposer que l'atelier à l'origine du thème du serpent dans une île soit à situer au nord du Valois. Ce dont j'ai déduit que cette création avait dû attendre la fin du Xviri ${ }^{\mathrm{e}}$ siècle, au moment où les écrits de Palissy ont été redécouverts et popularisés ${ }^{38}$, parallèlement à la découverte des couches géologiques du Cuisien (plus tardivement reconnues et décrites que celles du Lutétien) ${ }^{39}$. Or, l'identification récente de ce plat, dont le décor « au serpent dans une île » comporte un dragon sur l'aile, sur le catalogue illustré d'Amiens datant de la fin du XviI ${ }^{\mathrm{e}}$ ou du début du XVIII ${ }^{\mathrm{e}}$ siècle, permet de prouver l'ancienneté de cette production $^{40}$. Bien que le dessin ne comporte pas de coquilles identifiables, le choix et la répartition des animaux et surtout des feuillages permettent de souligner que l'ensemble attribué par L. Amico à son « Maître du griffon » doit être fortement élargi. Ce dessin renforce en particulier l'intérêt chronologique du plat du Louvre MR 3529, provenant de Cassel (voir fig. 1 a et b du Corpus des céramiques post-palisséennes dans ce volume), qui appartient au même groupe et que l'on connaît par des tirages présents dans d'autres collections ${ }^{41}$.

En revanche, le plat ovale du Louvre MR 3530, provenant de Brunswick (voir fig. 2 a et b du Corpus des céramiques post-palisséennes, dans ce volume), semble être le représentant d'un autre type de création. Le groupement de trois coques au centre d'un cercle de petits gastéropodes, à la place des habituels reptiles axiaux dans l'île, qui se retrouve sur d'autres plats ovales ou ronds ${ }^{42}$, peut aussi être remplacé par une couleuvre en boucle ${ }^{43}$, et il est également associé au brocheton, au chabot, aux grenouilles tachetées de noir et à la source jaillissante. Les coquilles fossiles et actuelles diffèrent cependant significativement de celles de l'ensemble au dragon (à l'exception d'une petite patelle qui pourrait être caractéristique). Le Clavilithes et les turritelles fossiles sont assurément d'origine cuisienne, mais il manque les potamides typiques de Cuise. Les insectes sont uniquement des papillons modelés et les végétaux diffèrent aussi dans le détail. C'est donc un bon exemple de la difficulté d'identifier un sousensemble distinct, en raison d'affinités certaines avec l'ensemble des plats rattachés à l'atelier du « Maître du dragon ».

Le petit plat ovale conservé à Rennes 794.1.569 (voir fig. 20 a et b du Corpus des céramiques post-palisséennes, dans ce volume), qui présente les potamides typiques du Cuisien, avait été décrit par Amico à partir d'une pièce identique du British Museum (1883.1019.12). Il se rattache au groupe des plats présentant un décor de graviers dans l'eau ${ }^{44}$. 


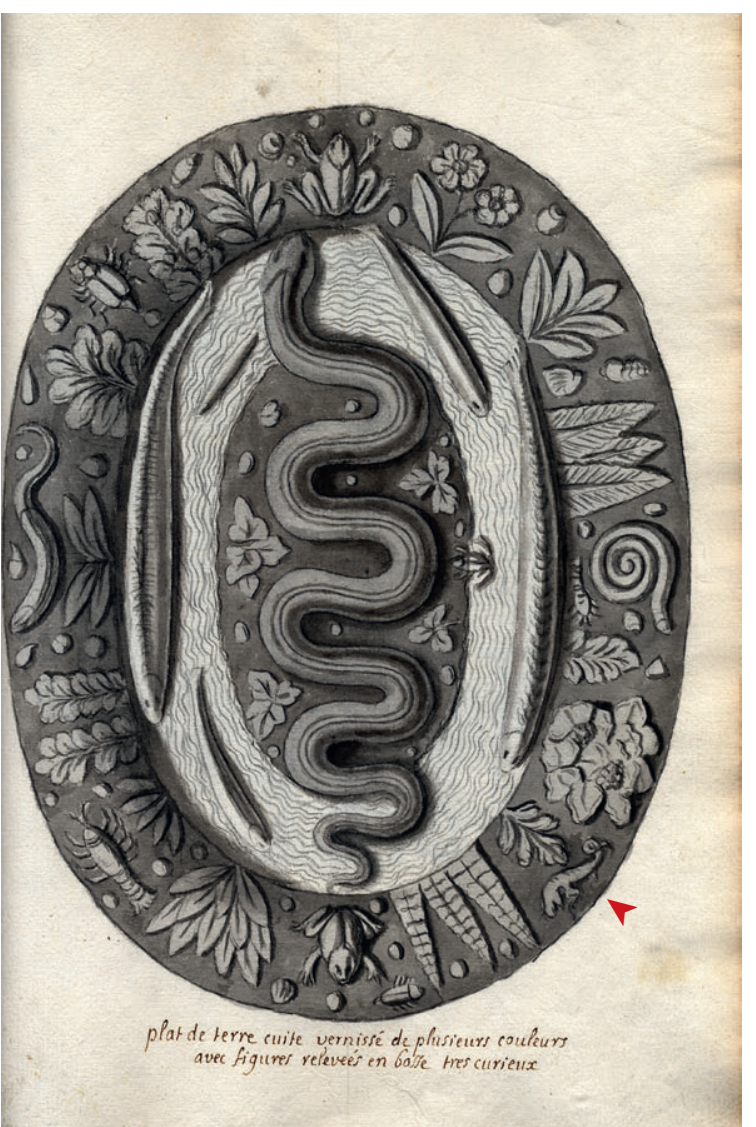

Fig. 18. Étienne de Faye (1670-1750), Dessin à l'encre de Chine d'un Plat à décor rustique (qui comporte une représentation maladroite du « dragon ", en bas à droite), dans Description d'un cabinet et d'un médaillier, entre 1690 et 1738, Amiens, Bibliothèques d'Amiens-Métropole, MS $400 \mathrm{E}$, pl. 301. C Cliché Bibliothèques d'Amiens-Métropole.

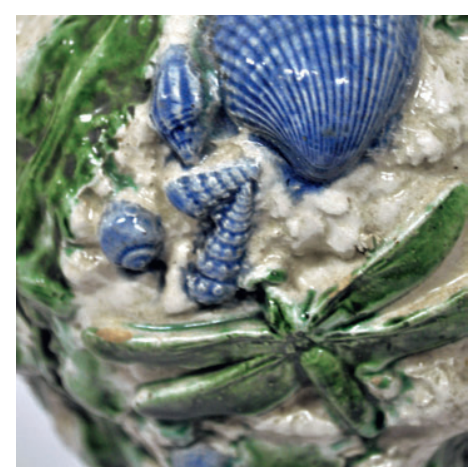

Fig. 19. Céramique post-palisséenne : Venericor (grand bivalve) et potamides, Ampullariidae et volute fossiles, associés à la libellule modelée, Rennes, musée des BeauxArts, 794.1.570. (C) J.-C. Plaziat.
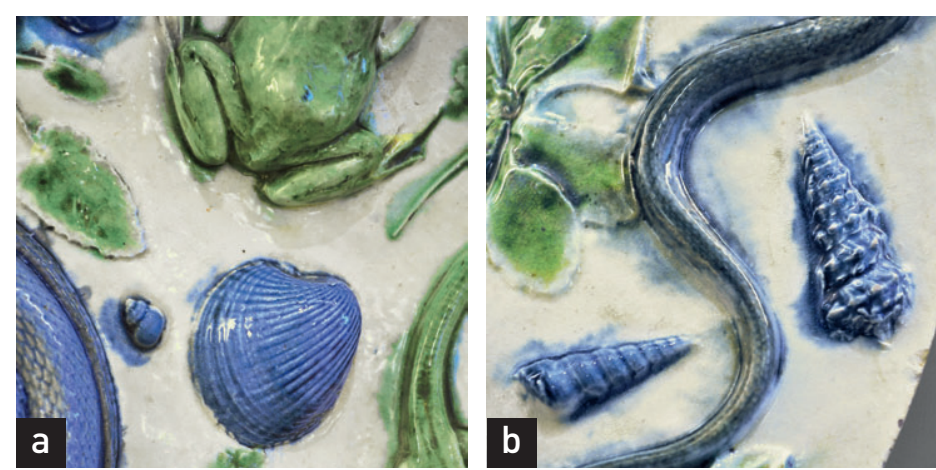

Fig. 20. Céramique post-palisséenne : a. Venericor et Batillariidé de l'Éocène, associés à une Turritelle (b) qui peut être actuelle, Sèvres, musée national de Céramique, MNC 3145. (c) J.-C. Plaziat.
C'est une autre pièce clé, superbe variation autour du thème du serpent axial, ici une couleuvre en boucle, sans anneau aquatique ou comportant une couleuvre dépassant de l'île, avec des écrevisses particulièrement grandes et des moulages extrêmement fidèles de coquilles fossiles, dont ceux des potamides et du Clavilithes caractéristiques du Cuisien de Cuise. Les insectes (papillons, mouche ou abeille) sont modelés, et les moulages de végétaux plus diversifiés, dont la répartition est moins rigide, contribuent à la définition d'un autre sousensemble particulièrement imaginatif et bien réalisé ${ }^{45}$.

De même, le petit plat ovale du Louvre MR 3543, avec un lézard sur fond bleu lisse, qui provient de la collection de Brunswick (voir fig. 3 a et b du Corpus des céramiques postpalisséennes, dans ce volume), paraît isolé par plus d'une particularité. Le lézard pourtant moulé comporte une queue ondulée, étonnamment irréaliste, et les coquilles actuelles principales (trois pétoncles) n'ont pas d'équivalent dans le corpus post-palisséen. Les trois gastéropodes fossiles sont bien du Tertiaire parisien, mais ne sont pas typiques du Cuisien. Le décor de cette pièce n'est donc guère rattachable à une autre création connue.

Il est aussi difficile de traiter des pièces de forme de référence (pichets, aiguières, fontaines de table à décor rustique), que l'on doit cependant situer dans l'ensemble post-palisséen lorsqu'elles comportent des moulages de fossiles. Leurs décors sont particulièrement disparates et la qualité des moulages de coquilles laisse souvent à désirer, l'identification des fossiles au niveau de l'espèce devenant même souvent impossible, bien qu'il s'agisse uniquement de formes de l'Éocène parisien.

Les fossiles de l'aiguière de Rennes (voir fig. 3 de l'article de P. Lehuédé et al. dans ce volume et fig. 19) suggèrent un rapprochement avec les plats du musée de Sèvres MNC 3145 (voir fig. 5 de l'article de P. Lehuédé et al. dans ce volume) et du Getty Museum 88 DE $63^{46}$, en raison des excellents moulages de fossiles (la Venericor et les Batillariidés), colorés en bleu sur fond blanc comme sur le plat de Sèvres (fig. 20). L'association avec le Clavilithes de Cuise conduit à envisager une certaine proximité avec les plats ornés de potamides de Cuise, nécessairement antérieurs à la Révolution, comme le plat de Rennes 794.1.569.

Enfin, la fontaine de table en forme de rocher surmonté d'une statuette de joueur de cornemuse, qui est conservée au musée de Picardie et figure sur le manuscrit d'Amiens (voir fig. 2 et 3 de l'article de F. Barbe et al. dans ce volume), paraît plus isolée, bien qu'elle soit très proche d'une autre fontaine de table conservée au Victoria and Albert Museum ${ }^{47}$. Malgré la très grande imperfection des moulages, il a été possible d'identifier sur la pièce d'Amiens, dans l'assemblage de coquilles actuelles et fossiles moulées, la turritelle, le Clavilithes et des potamides du Cuisien avec d'autres fossiles du Lutétien. On y trouve aussi la seule occurrence connue de Tympanotonos fuscatus, une grande espèce actuelle de potamide qui caractérise les estuaires et lagunes d'Afrique occidentale (fig. 21) ${ }^{48}$. 


\section{Annexe. Tableau des espèces}

Identification scientifique des animaux et plantes moulés sur nature, dans l'ordre systématique : pour les fossiles, d'après Cossmann et Pissaro (1904-1913), dont la systématique a été réactualisée par Pacaud et Le Renard (1995).

Leur distribution en fonction des différents ensembles de pièces à décor rustique étudiées est indiquée sans indice de fréquence :

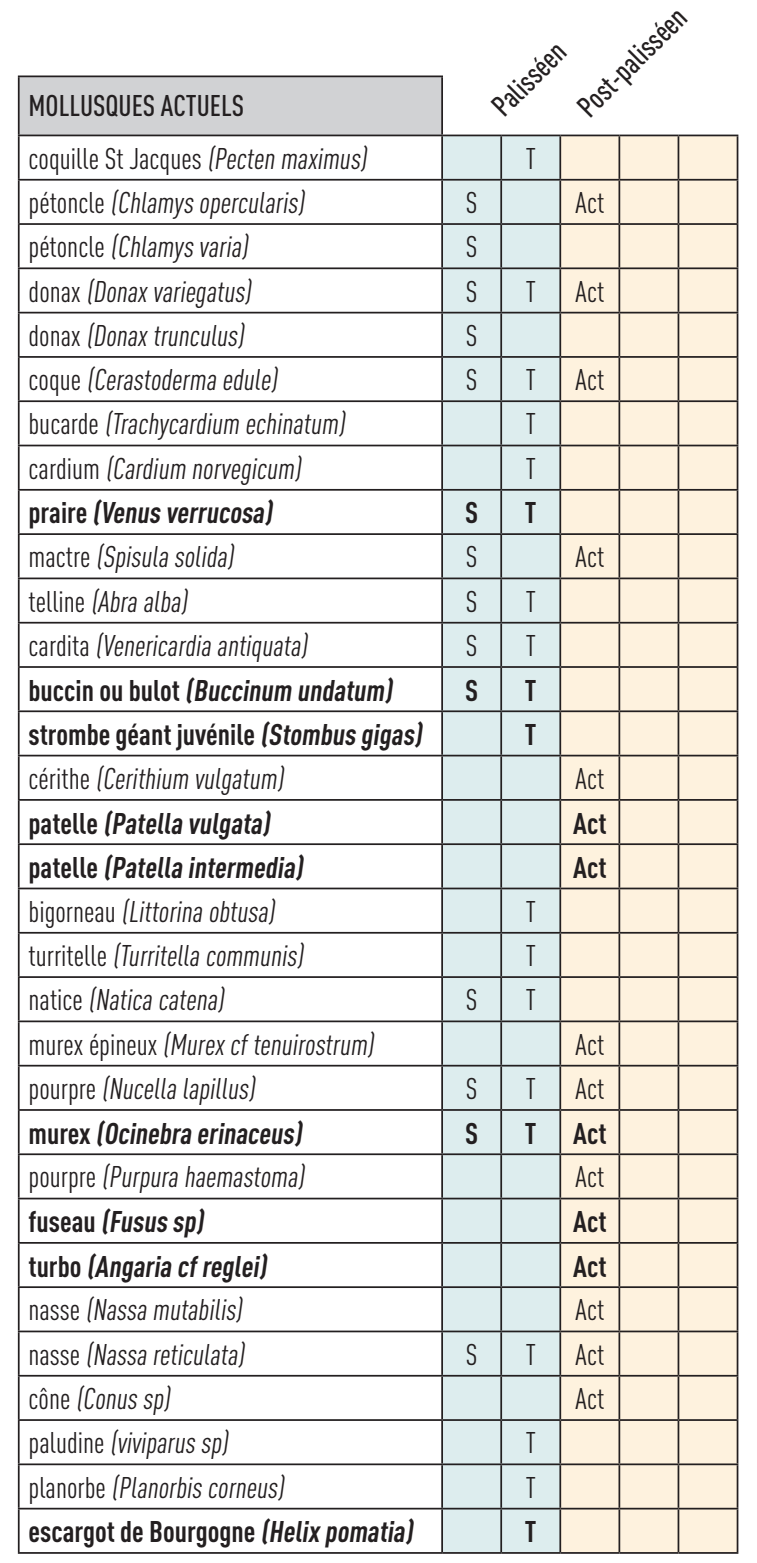

Ensemble palisséen : S - vaisselle d'apparat de la « façon de Saintes "; T - pièces extraites des fouilles de l'atelier des Tuileries.

Ensemble post-palisséen : Act - mollusques actuels ; C - fossiles du Cuisien ; L - fossiles du Lutétien ; C-L - fossiles communs au Cuisien et au Lutétien.

En gras : les éléments les plus caractéristiques du point de vue typologique. En rouge, les fossiles cuisiens du faciès de Cuisela-Motte (Oise).

\begin{tabular}{|c|c|c|c|c|c|}
\hline AUTRES INVERTÉBRÉS ET VERTÉBRÉS & & & & & \\
\hline $\begin{array}{l}\text { hanneton de juin modelé en partie } \\
\text { ou en totalité }\end{array}$ & & & Act & & \\
\hline papillon modelé & & & Act & & \\
\hline chenille de grande taille & & & Act & & \\
\hline $\begin{array}{l}\text { libellule aux ailes modelées: } \\
\text { à nervures imaginaires }\end{array}$ & & & Act & & \\
\hline longicorne modelé & & & Act & & \\
\hline $\begin{array}{l}\text { crabe enragé ou crabe vert } \\
\text { (Carcinus moenas) }\end{array}$ & $S$ & & & & \\
\hline écrevisse à pattes rouges (Astacus astacus) & S & $\mathrm{T}$ & Act & & \\
\hline grondin gris (Eutrigla gunardus) & S & & & & \\
\hline raie (Raja undulata ou brachycera) & $S$ & & & & \\
\hline perche (Perca fluviatilis) & & & Act & & \\
\hline ablette (?) (Alburnus alburnus) & & & Act & & \\
\hline brochet (très jeune) (Esox lucius) & & & Act & & \\
\hline gardon (Rutilus rutilus) & & & Act & & \\
\hline goujon (Gobio gobio) & & & Act & & \\
\hline chabot (Cottus gobio) & & & Act & & \\
\hline grenouille verte (Rana esculenta) & $\mathrm{S}$ & $\mathrm{T}$ & Act & & \\
\hline rainette (?) (Hyla sp) & $\mathrm{S}$ & & & & \\
\hline $\begin{array}{l}\text { cistude d'Europe, tortue d'eau } \\
\text { (Emys orbicularis) }\end{array}$ & S & & & & \\
\hline lézard vert (Lacerta viridis) & S & $\mathrm{T}$ & Act & & \\
\hline lézard des murailles (Podarcis muralis) & $S$ & & & & \\
\hline couleuvre à collier (Natrix natrix) & $S$ & $\mathrm{~T}$ & Act & & \\
\hline vipère aspic (Vipera aspis) & $\mathrm{S}$ & & & & \\
\hline
\end{tabular}




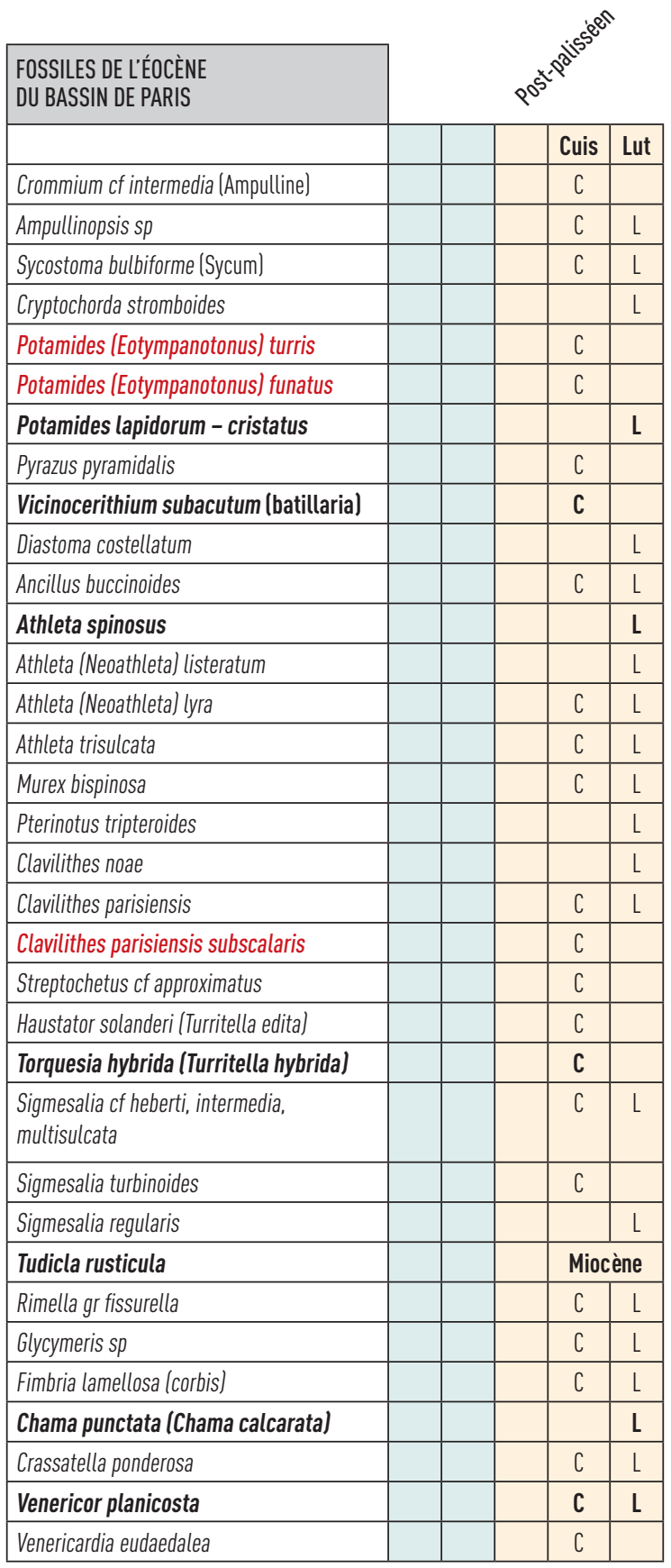

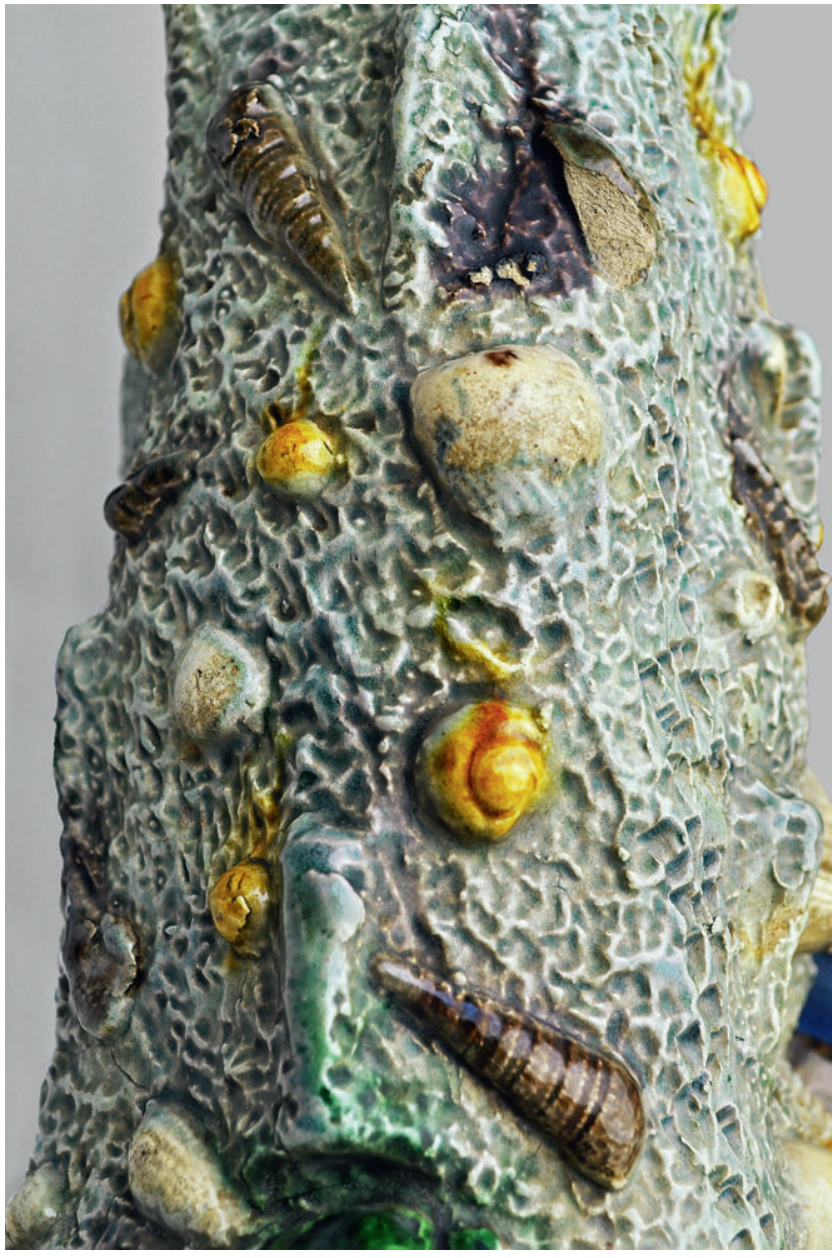

Fig. 21. Céramique post-palisséenne : Tympanotonos fuscatus (seule figuration connue de cette espèce africaine actuelle), associe à des fossiles de l'Éocène (mal moulés), Fontaine de table à décor rustique, Amiens, musée de Picardie, MP 1876-304 bis. (c) C2RMF/A. Maigret. 


\section{Conclusion}

Cette approche typologique naturaliste, qui va au-delà de l'identification zoologique, botanique et paléontologique du décor rustique moulé, permet d'ébaucher une méthode de regroupement des pièces examinées dans les musées et sur des documents iconographiques fiables, qui semble plus rationnelle que les propositions antérieures. Elle doit encore être approfondie et confrontée notamment aux résultats des analyses physico-chimiques, ce que tentera de mener à bien le projet de recherche Figulines ${ }^{49}$.

Bien qu'il soit prématuré de proposer une distinction d'ateliers à l'intérieur de l'ensemble post-palisséen, on peut dorénavant l'envisager avec vraisemblance, au vu du croisement entre les regroupements typologiques fondés sur l'identification des fossiles et la provenance des pièces historiques de référence. Une production en Île-de-France, à partir du $\mathrm{XVII}^{\mathrm{e}}$ siècle, dans un petit nombre d'ateliers, est une hypothèse vraisemblable. Car il est peu probable que les modestes fossiles utilisés aient été fournis à partir de ce qu'on appelle les cabinets de curiosités, constitués par achats, dans lesquels seuls les coquillages spectaculaires et exotiques pouvaient trouver place. À l'époque de la production de la vaisselle rustique post-palisséenne, la récolte des coquilles fossiles, en particulier celles du Valois, a donc dû être effectuée par un familier de ces dépôts fossilifères plus ou moins éloignés de la capitale (près de Pierrefonds) : peut-être un fournisseur de matière première minérale (par exemple des sables fins associés au Cuisien fossilifère, propices à la verrerie et au moulage, comme les sables de Saint-Gobain), en relation avec des ateliers de céramique?

Le décor des rustiques figulines post-palisséennes comporte des fossiles tout à fait étrangers aux créations de Palissy à Saintes, même s'il a eu l'intention d'illustrer ses conceptions naturalistes pré-scientifiques, diffusées publiquement (oralement à Paris à partir de 1575) et publiées en $1580^{50}$. Mais, étant donné l'absence de pièces authentiques accessibles au simple artisan d'art excluant une imitation ou une réelle filiation, jusqu'à quel point peut-on dire que cette vaisselle, postérieure de plusieurs décennies à celle du maître, constitue un hommage purement intellectuel à sa démarche «naturaliste »? L'auteur du premier plat au serpent dans une île a-t-il eu connaissance de la curiosité (tardive) de Palissy pour les fossiles du Tertiaire parisien à travers la lecture de son œuvre majeure (de 1580) ? Combien d'ateliers ont-ils poursuivi cette tradition de moulage sur nature d'éléments rustiques ? L'existence d'ateliers concurrents expliquerait-elle l'apparition de surmoulages-pirates des créations?

Fruit de longues années de recherche en amateur, ce travail tente de combiner l'exigence scientifique des identifications taxinomiques à des hypothèses sur la production de Palissy et des artisans-créateurs post-palisséens. Les nombreuses questions qu'il soulève ont rencontré celles des historiens de l'art et n'ont pas d'autre ambition que de proposer un éclairage naturaliste à des recherches devenues nécessairement pluridisciplinaires.

\section{Notes}

1. Réunis pour la première fois de façon critique par Cap, 1844 (lui-même réédité à l'identique, en 1961, avec une préface de J. Orcel) et modifiant la réédition complète mais contestable de Faujas de Saint-Fond et Gobet, 1777. Des rééditions plus récentes, partielles ou complètes mais sans nouveauté, peuvent être aussi consultées utilement, notamment l'édition sous la direction de M.-M. Fragonard, 2010.

2. A la suite d'un retour aux textes initié par des historiens de la géologie, dans le cadre du Comité Français pour l'Histoire de la Géologie (COFRHIGEO), Ellenberger, 1988 ; Plaziat, 2009, 2010, 2011.

3. Brongniart, 1844 ; Orcel, 1961 ; Plaziat in Ellenberger, 1988 ; Plaziat, 1997.

4. Elle remarque cependant leur distribution sur les céramiques et fait référence à l'ouvrage d'Ellenberger, 1988, où ils sont pris en compte pour la première fois ; Perrin, 2001 (publication de la thèse de 1998).

5. Ces nouveaux résultats, après rectification des identifications paléontologiques erronées de Brongniart, avaient jusqu'alors été réservés aux géologues et aux historiens régionaux dans Plaziat, 2009, 2010, 2011, ou plus largement : Vacher, Plaziat, 2010 ; Barbe, 2010.
6. Palissy, La Recepte Véritable, dans Palissy, 2010, p. 166.

7. Bénéficiaire d'un des premiers « bassins rustiques ", estimé à 50 écus en 1556 (Palissy, 1563, dans Palissy, 2010, p. 53-54).

8. Inventaires de 1556, 1559, 1568 (Amico, 1996, p. 229 et 231) : «semée de coquilles et plusieurs animaulx. » Cependant, sans référence nominale à Palissy.

9. Kris, 1926 ; Amico, 1996, p. 86. 10. Palissy, Architecture et ordonnance de la grotte rustique, dans Palissy, 2010, p. 76.

11. Ce dont J. Héroard témoigne peutêtre, avant 1610, en s'offusquant de voir l'enfant Louis (futur Louis XIII) jouer avec l'une de ces précieuses " antiquailles », Foisil, 1989.

12. Pour le décor d'insectes, de miniserpents ou d'éléments divers (dragon, nid d'oiseau) qui font plutôt penser à des moulages de pièces d'orfèvrerie.

13. Pour un inventaire récent, illustré et référencé, voir Katz, Lehr, 1996 ; Viennet, Starosta, 2010

14. Cardiidés des genre Cerastoderma et Trachycardium.

15. Chlamys.

16. Venus.
17. Dans ce tableau, les dénominations naturalistes, selon une systématique scientifique actualisée, sont mises en relation avec les appellations populaires.

18. Voir Barbe, Bouquillon, 2010.

19. On parle aussi de nœuds de serpents, bien qu'il s'agisse chaque fois d'un seul individu disposé en une masse compacte. La forme lovée en spirale est réservée à des moulages de couleuvres juvéniles. Mais sur les céramiques postpalisséennes, cette dernière disposition correspond à un modelage.

20. Que l'on observe sur ses diverses créations, comme sur le plat du Louvre MR 2337, mais aussi sur les briques glaçurées des Tuileries.

21. Voir l'article de A. Gerbier dans ce volume.

22. Clémens, 1991, Amico, 1996, fig. 100 pour la guêpe (Écouen, musée national de la Renaissance EP 1594) et Amico, 1996, fig. 12, pour le phoque entreposé aussi avec soin près du four des Tuileries, Écouen, musée national de la Renaissance, EP 2696 (Dufaÿ et al., 1987, fig. 25).

23. Notons cependant une coquille juvénile, de moins de $10 \mathrm{~cm}$, figurée sans commentaire par Amico, 1996, fig. 4. 
24. Une centaine de pièces, mais correspondant à quelques dizaines de modèles en raison de tirages multiples et d'éventuels surmoulages.

25. Souvent MR 2293 du musée du Louvre.

26. Voir l'article de J. Denis-Dupuis dans ce volume et Dupuis, 2016.

27. Voir l'article de F. Barbe et al. dans ce volume.

28. A. Brongniart en premier, dans son Traité des arts céramiques, 1844. Mais l'intérêt de ces fossiles n'a été explicité que très tardivement, car il ne pouvait pas être mis en évidence sans les connaissances paléontologiques régionales acquises ultérieurement (Plaziat, 1997, 2009, 2010 , 2011)

29. Principalement dans des assemblages originaux comme ceux du musée de Sèvres, MNC 3145, et du musée Paul Getty, 88 DE 63, sans étendue d'eau, et celui du musée de Varsovie, SZC 1640, également sans île.

30. Illustrations dans Ellenberger, 1988 et Plaziat, 2011

31. Dont les plus caractéristiques sont le potamide Eotympanotonus turris et le batillariidé Vicinocerithium subacutum. 32. Clavilithes parisiensis subscalaris, Turritella edita.

33. Amico, 1996, p. 128 et fig. 110, 111. Par la suite, I. Perrin a précisé l'attribution faite par L. Amico en distinguant des ateliers différents : celui du « Maître du griffon " et celui " aux traces de séparateurs cylindriques », Perrin, 2001 p. 253.

34. Par exemple, les plats du Louvre MR 2293 et OA 1358, et celui du British Museum 1883.10-19.12, figurés par Amico, 1996, fig. 96-105

35. Comme le plat du Louvre MR 2294, Amico, 1996, fig. 107.

36. Par exemple, les plats du Louvre OA 1358, de la Wallace collection C 173, du Victoria \& Albert Museum 5476-1859 et du Metropolitan Museum de New York 53-225-39.

37. Plat MR 3530, au décor coquillier de l'île si particulier (Amico, 1996, fig. 108) et MR 3529 déposé à Écouen (E.Cl. 13202).

38. A partir des commentaires de Fontenelle (1720) et de la réédition (fâcheusement remaniée) par Faujas de Saint-Fond et Gobet, 1777.

39. Plaziat, 2009.

40. Voir l'article de F. Barbe et al. dans ce volume.

41. Comme les plats d'Écouen

E.Cl. 1141 et de Cleveland 69-106.

42. Gibbon, 1986, fig. 32.

43. Comme sur les plats du Louvre

MR 2294 et du château d'Anet 483.

44. Comme sur le plat de la Wallace Collection (C 173) et celui du Fitzwilliam Museum, en faïence et daté de 1638. Amico, 1996 , p. 120, fig. 102-104

45. Que l'on retrouve sur d'autres plats comme ceux du Louvre MR 2293 et

d'Écouen E.Cl. 1142.

46. Qu'Amico avait attribués à Palissy : Amico, 1996, p. 101.
47. Victoria \& Albert Museum 537-1865.

48. Ce qui n'est pas le cas sur

le rocher du Victoria \& Albert Museum qui semble pourtant provenir du même atelier.

49. Sur ce projet de recherche, voir

l'introduction de ce volume.

50. Par ses conférences publiques

parisiennes et l'ouvrage publié qui en est explicitement le développement, Palissy,

1580 (dans Palissy, 2010)

\section{Bibliographie}

Amico L., 1987, « Les céramiques rustiques authentiques de Bernard Palissy », Revue de l'Art, $\mathrm{n}^{\circ}$ 78, p. 61-69.

Amico L., 1996, A la recherche du paradis terrestre. Bernard Palissy et ses continuateurs, Flammarion, Paris.

Barbe F., 2010, « Le cinq centième anniversaire de la naissance de Bernard Palissy. Coquillages ou fossiles : du nouveau sur les "rustiques figulines" ", Bulletin monumental, 168, p. 29-30.

Barbe F., Bouquillon A., 2010, « À la redécouverte des créations de Bernard Palissy : l'aiguière et le plat à décor de "rustiques figulines" du musée du Louvre ", La Revue des musées de France, Revue du Louvre, $\mathrm{n}^{\circ}$ 5, p. 24-33.

Brongniart A., 1844, Traité des arts céramiques ou Des poteries considérées dans leur histoire, leur pratique et leur théorie, 2 tomes, Béchet Jeune, Paris (troisième édition, 1877).

Cap P.-A. (éd.), Palissy B., 1844, Euvres complètes de Bernard Palissy, J.-J. Dubochet et Cie, Paris. Réédition, 1961, Librairie Scientifique A. Blanchard, Paris.

Clémens J., 1991, « Fossalou guêpe de Bernard Palissy ", Garona, Bordeaux, p. 141-145.

Dufaÿ B., de Kisch Y., Poulain D. Roumégoux Y., Trombetta P. J., 1987, "L'atelier parisien de Bernard Palissy ", Revue de l'Art, n ${ }^{\circ} 78$, p. 33-60.

Dupuis J., 2016, « La céramique dite "d'Avon" : retour historiographique et mise au jour d'une attribution légendaire ", Revue de l'Art, n 193/20163 , p. $27-34$

Ellenberger F., 1988, Histoire de la géologie, I : Des Anciens à la première moitié $d u X_{V I I}^{e}$ siècle, Lavoisier, Paris.

Faujas de Saint-Fond B., Gobet N., 1777, Euvres de Bernard Palissy, revues sur les exemplaires de la bibliothèque du roi (...), Ruault, Paris.

Foisil M., 1989, Journal de Jean Héroard, médecin de Louis XIII (1638), Fayard, Paris

Gibbon A., 1986, Céramiques de Bernard Palissy, Librairie Séguier/ Vagabondages, Éditions GaramontArchimbaud/Birr, Paris.

Katz M. P., Lehr R., 1996, Palissy ware. Nineteenth-century French Ceramists from Avisseau to Renoleau, The Athlone Press, Londres.
Kris E., 1926, Le style rustique, le moulage d'après nature chez Wenzel Jamnitzer et Bernard Palissy. Réédition, 2005, postface de P. Falguières, «Sur le renversement du maniérisme », Macula, Paris.

Orcel J., 1961, « Préface au nouveau tirage des Cuvres complètes de Bernard Palissy éditées par Cap ", Librairie Scientifique A. Blanchard, Paris, p. 1-17.

Palissy B., 2010, Euvres complètes, Fragonard M.-M. (dir.), seconde édition revue et annotée, Honoré Champion, Paris.

Perrin I., 2001, Les techniques céramiques de Bernard Palissy, thèse sous la direction de M. Jean Guillaume, soutenue en 1998, université Paris IV Sorbonne, Anrt, Lille, 2 vol.

Plaziat J.-C., 1977, « Les Cérithidés tropicaux et leur polymorphisme lié à l'écologie littorale des mangroves ", Malacologia, 16 , p. 35-44.

Plaziat J.-C., 1997, « L'importance des coquilles fossiles du Tertiaire parisien dans l'œuvre scientifique et artistique de Bernard Palissy, à la fin du seizième siècle », dans Gohau G. (éd.), De la géologie à son histoire, ouvrage édité en hommage à François Ellenberger, Comité des Travaux Historiques et Scientifiques, Paris, p. 15-24.

Plaziat J.-C., 2009, « Bernard Palissy à Venteuil en Champagne : un épisode essentiel d'une biographie démythifiée. La montagne de Reims depuis l'origine de la paléontologie et de la géologie françaises (1580-2007) ", Études marnaises (Bull. de la SACSAM), tome 124, p. 53-111.

Plaziat J.-C., 2010, « Bernard Palissy (1510$1590)$, un authentique géologue ? Près de 500 ans d'incompréhension, entre oublis et réhabilitation au premier rang des précurseurs de la géologie », Géochronique, $\mathrm{n}^{\circ} 115$, p. 11-15.

Plaziat J.-C., 2011, "Bernard Palissy (15101590) and the French geologists: a critical reappraisal concerning the founding naturalist and his rustic ceramics", Bulletin de la Société géologique de France, vol. 182, n 3, p. 255-267.

Poulain D., 1993, "Les "rustiques figulines" du musée des Beaux-Arts de Lyon ", Bulletin des Musées et Monuments lyonnais, vol. 3-4, p. 24-47.

Vacher C., Plaziat J.-C., 2010, Bernard Palissy entre les lignes. L'homme, l'artiste, le savant, à travers cinq siècles d'écrits. Plaquette en vente au musée Bernard-Palissy, Lacapelle-Biron.

Viennet C., Starosta P., 2010, Bernard Palissy et ses suiveurs $d u X_{V I}^{e}$ siècle à nos jours. Hymne à la nature, Faton, Dijon. 